$$
\begin{aligned}
& \text { 震 }
\end{aligned}
$$

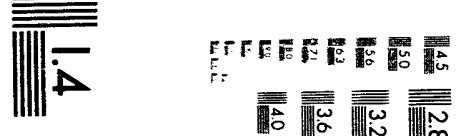

$$
\begin{aligned}
& \text { 雴市 }
\end{aligned}
$$



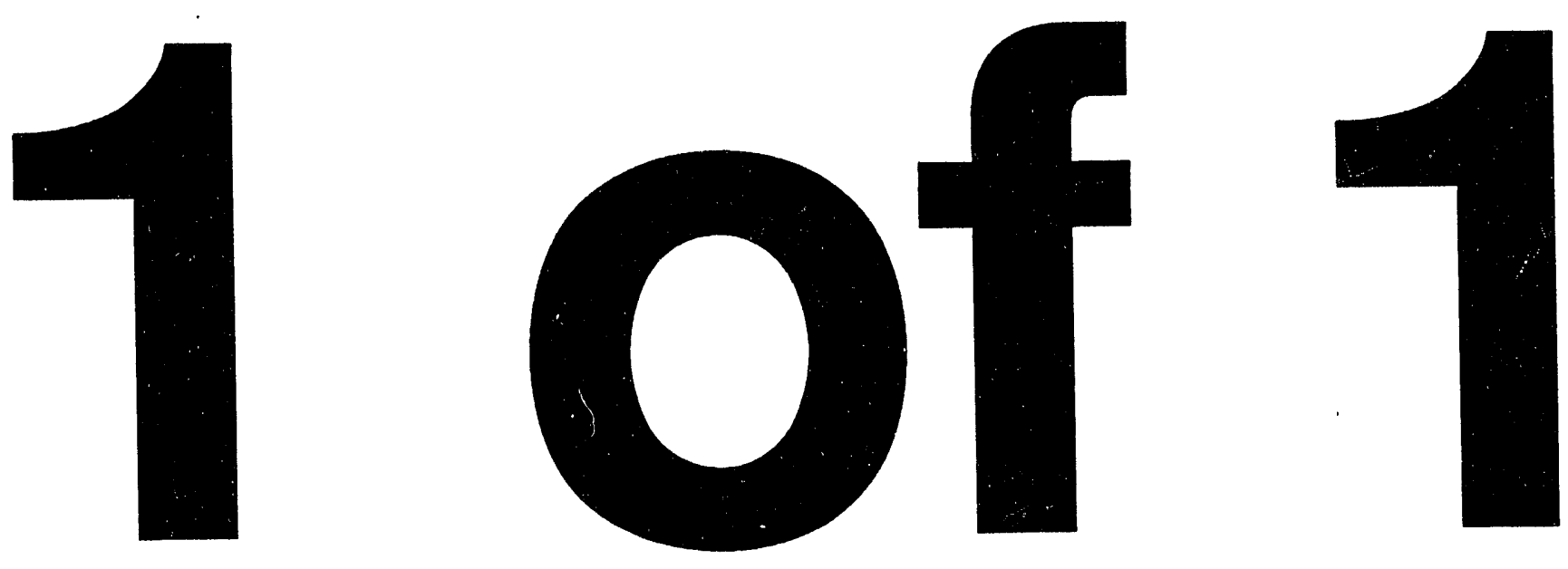


\title{
NUMERICAL EVALUATION OF HIGH ENERGY PARTICLE EFFECTS IN MAGNETOHYDRODYNAMICS
}

\author{
R. B. White and Yanlin Wu \\ Plasma Physics Laboratory, Princeton University, P.O.Box 451, Princeton \\ NJ 08543 USA
}

\begin{abstract}
The interaction of high energy ions with magnetohydrodynamic modes is analyzed. A numerical code is developed which evaluates the contribution of the high energy particles to mode stability using orbit averaging of motion in either analytic or numerically generated equilibria through Hamiltonian guiding center equations. A dispersion relation is then used to evaluate the effect of the particles on the linear mode. Generic behavior of the solutions of the dispersion relation is discussed and dominant contributions of different components of the particle distribution function are identified. Numerical convergence of Monte-Carlo simulations is analyzed. The resulting code ORBIT provides an accurate means of comparing experimental results with the predictions of kinetic magnetohydrodynamics. The method can be extended to include self consistent modification of the particle orbits by the mode, and hence the full nonlinear dynamics of the coupled system.
\end{abstract}




\section{Introduction}

The interaction of high energy particles with magnetohydrodynamic modes is of great importance for controlled thermonuclear fusion. The principal effects are the stabilization of the sawtooth ${ }^{1-7}$ due to the rigidity of the plasma provided by the precessing trapped particles, and the destabilization of other modes ${ }^{8-14}$ through resonance with either trapped or passing particles with the possible resultant loss of a large fraction of them. ${ }^{15,16}$ The basic theoretical treatment involves the separation of the plasma into two parts, with the background plasma described with fluid magnetohydrodynamics (MHD) and the high energy component described with gyrokinetic or guiding center equations. Present experiments reporting these effects involve high energy particles produced through neutral beam injection or heating at the ion cyclotron frequency. ${ }^{17,18}$

It is important that careful analysis of these experiments be made in order to correctly predict the possible effects of fusion-generated alpha particles on the same modes. Present estimates for ignited plasmas indicate that the fusion alpha particle density is marginal for sawtooth stabilization and fishbone destabilization. Of particular interest is the investigation of the sawtooth-free domain of tokamak operation. This stable domain has several properties which make it of interest for controlled thermonuclear fusion. It is a high beta domain. The energetic particles can be chosen so that the upper bound of the domain is near the Troyon limit or even higher if the device has access to the second stability domain. It is a high current domain. With no sawtooth, a tokamak could be operated with a safety factor $q(r)$ having a value of 0.5 at the magnetic axis. This low value of $q(0)$ would imply fairly high shear, and hence improved stability to other magnetohydrodynamic modes such as the resistive instabilities and the major disruption. There is a stable window of operation in the plane of high energy particle density and background plasma beta which increases in size with plasma temperature and thus has good reactor application potential.

Besides these beneficial effects, the investigation of mode destabilization and induced particle loss is of considerable importance. Significant induced alpha particle loss could cause problems through excessive wall loading and loss of ignition margin.

Previous treatments either include a complete analysis of both the MHD 
and gyrokinetic components of the plasma, and are overwhelmed by the computing requirements, which do not allow a reasonable study of the dependence of the results on the plasma parameters ${ }^{19}$, or they suffer from several approximations, and although they show a rough agreement with experiments, a detailed comparison is not fully warranted. A major impediment is the use of analytic equilibria. The resonant interaction of the particles with the modes depends on the toroidal precession rate. Even in codes using numerical equilibria,${ }^{20}$ analytic expressions for the precession ignore the important effects of plasma shape, the possible presence of a magnetic well, and plasma rotation, all of which can significantly modify the precession rate. In addition, although it is justified to carry out a large aspect ratio expansion in the derivation of the dispersion relation, it may not be reasonable to neglect the interaction of the particles with higher poloidal harmonics.

The usual lowest order expansion keeps only the first poloidal $(m=1)$ harmonic $\xi_{1}$, and while this is a good approximation in the center of the plasma where the safety factor $q<1$, it neglects the interaction of the mode with all particles outside this domain. Numerical simulations of particle loss and energy exchange during the fishbone mode ${ }^{15}$ indicate that the $\mathrm{m}=2$ harmonic plays an important role in this process, particularly because its contribution is in a domain where $\xi_{1}$ is zero. The $\mathrm{m}=3$ harmonic can contribute to particle loss by resonant expulsion of particles near the plasma edge, but mode-particle energy exchange and hence contribution to mode destabilization is normally negligible. Finally, the particle distributions are often idealized, and only approximately represent the high energy particle distributions present in the experiments.

In this work we describe an analysis which corrects these problems. Excessive computing requirements are avoided by considering the plasma on a time scale which is short with respect to changes in the equilibrium profile. In addition, mode-mode coupling effects are ignored. In the case of the modes considered this is a very reasonable approximation, the dominant destabilization and saturation mechanisms are due to the mode-particle interaction. Thus the magnetohydrodynamic state of the plasma is given by parameters describing the current and pressure profiles, and by the solution of the Grad-Shafranov equation for the equilibrium to be considered. For some modes (sawtooth, fishbone) the ordering of the high energy particle effects $^{11}$ guarantees that the form of the mode is not greatly modified by 
the high energy particles, and an ideal MHD code can be used to determine the eigenfunctions, but for other modes, the kinetic terms are important in determining the shape of the mode and a code such as NOVA- $\mathrm{K}^{20}$ must be used to determine the mode spectrum and shape.

Were it not for the presence of the singular dissipative layer (either inertial or resistive, depending on plasma parameters) it would be possible to simulate the coupled system through energy conservation. Energy transferred to and from the particles through resonance with the mode is readily observed in guiding center simulations, and this rate of energy transfer could be used to calculate the mode growth rate. The presence of the dissipative layer, which transfers energy to the electrons through kinetic modes not treated in a simple MHD analysis, makes such a simple treatment incorrect, and requires that one instead model the dissipative processes or solve a dispersion relation which includes mode-particle energy exchange, the dissipation of the layer, and the MHD energy of the mode in order to find the frequency and growth rate of the mode. In this work we use the variationally derived dispersion relation to find the complex mode frequency. In future publications other means will be explored.

The kinetic contributions to the variational dispersion relation are expressed in general magnetic coordinates. The contribution of the interaction of the particles with higher harmonics is retained. The effects of plasma shape and $\beta$ (i.e. the modification of plasma shape from concentric circular flux surfaces and the outward shift of the surfaces due to plasma pressure) are all properly included. Next, the integrals over the particle distribution are carried out using a Monte-Carlo simulation, which allows a reasonable approximation of the actual distribution functions. Finally, the bounce orbit averaging for trapped and passing particles is carried out using a Hamiltonian guiding center code using either analytical or numerically generated equilibria. This correctly gives the toroidal precession rates in the finite beta, shaped equilibria as well as including nonlinear particle dynamics. In addition the averaging over drift orbits takes into account the finite banana width of the trapped orbits and the drift excursions of the passing particles. A radial electric field can be used to give the effects of plasma rotation.

The dispersion relation is then solved using the magnetohydrodynamic parameters associated with the equilibrium and the kinetic contributions obtained from the guiding center averages. The results can be plotted following 
the variation of any parameter which does not involve a significant modification of the equilibrium, i.e. variation of the high energy particle density, plasma resistivity, diamagnetic drift frequencies, etc. If the amplitude of the mode is kept small and fixed, one obtains an analysis of the stabilizingdestabilizing properties of the fixed particle distribution function used. If instead an unstable mode is allowed to grow to an amplitude sufficiently large to modify the particle distribution, a self-consistent time dependent solution can be obtained with the instantaneous particle distribution determining the mode frequency and growth rate.

In the foliowing sections we sketch the derivation of the dispersion relation, describe the Monte-Carlo procedure using the guiding center equations, and show some generic results for the internal kink and fishbone modes. Detailed comparison with experiments and further analyses of the effects of the plasma shape and beta, of plasma rotation, and of the form of the high energy particle distribution function, will be described in future publications. The same techniques can be applied to analyze the interaction of high energy particles with any MHD mode. A treatment of the toroidal Alfven eigenmode ${ }^{13}$ (TAE) will be given in a separate publication.

\section{Dispersion Relation}

We begin with the contravariant and covariant expressions for the equilibrium field ${ }^{21,22}$

$$
\begin{gathered}
\vec{B}=\vec{\nabla} \zeta \times \vec{\nabla} \Psi_{p}+q \vec{\nabla} \Psi_{p} \times \vec{\nabla} \theta, \\
\vec{B}=g \vec{\nabla} \zeta+I \vec{\nabla} \theta+\delta \vec{\nabla} \Psi_{p},
\end{gathered}
$$

with $\Psi_{p}$ the poloidal flux, $\theta$ the poloidal angle, and $\zeta$ the toroidal angle. The coordinate system is a straight field line one, i.e. $q\left(\Psi_{p}\right)$ (the safety factor) gives the local helicity of a field line $q=d \zeta / d \theta$. The poloidal angle is chosen so that the Jacobian $\mathcal{J}^{-1}=\vec{\nabla} \Psi_{p} \cdot(\vec{\nabla} \theta \times \vec{\nabla} \zeta)$ is given by $B^{2} \mathcal{J}=g q+I$ with the volume element given by $d^{3} x=\mathcal{J} d \Psi_{p} d \theta d \zeta$. The magnetic field strength $B\left(\Psi_{p}, \theta\right)$ is independent of the coordinate $\zeta$. In a large aspect ratio circular equilibrium with $B=1-r \cos (\theta)$ these function are given to two orders in inverse aspect ratio by $\Psi=r^{2} / 2, d \Psi / d \Psi_{p}=q\left(\Psi_{p}\right), \mathrm{g}=1, I=r^{2} / q$, 
$\delta=0, \mathcal{J}=q / B^{2}$, with the field normalized to its value on axis and distances normalized to the major radius.

The equilibrium or set of equilibria is obtained numerically for the plasma under consideration. The high energy particle contribution to the dispersion relation is obtained by following a Monte-Carlo distribution of particles using a Hamiltonian guiding center formalism described previously. ${ }^{23,22}$ The dispersion relation takes the form ${ }^{11,1} D(\omega)=0$ where

$$
D(\omega)=I+\delta W_{M H D}+\delta W_{r}+\delta W_{n},
$$

with I the contribution from the inertial (resistive) layer, ${ }^{24}$

$$
\hat{I}=-\frac{8 i \Gamma\left(\left(\Lambda^{3 / 2}+5\right) / 4\right) \sqrt{\omega\left(\omega-\omega_{* i}\right)}}{\Lambda^{9 / 4} \Gamma\left(\left(\Lambda^{3 / 2}-1\right) / 4\right) \omega_{A}}
$$

and

$$
\Lambda=-i\left(\omega\left(\omega-\omega_{* e}\right)\left(\omega-\omega_{* i}\right)\right)^{1 / 3} / \gamma_{R}
$$

and the resistive growth rate $\gamma_{R}=S^{-1 / 3} \omega_{A}$. The diamagnetic frequencies are given by $\omega_{* e}$ and $\omega_{* i}$ and $S$ is the ratio of the resistive time to the Alfven time, all evaluated at the $\mathrm{q}=1$ surface. The term $\delta W_{M H D}$ is the usual fluid MHD contribution ${ }^{25} \delta \hat{W}_{M H D}=-\gamma_{I} / \omega_{A}$ with $\gamma_{I}$ referring to the ideal growth rate, and $\omega_{A}$ the shear Alfven frequency, $\omega_{A}=V_{A} /\left(\sqrt{3} R r_{s} q^{\prime}\right)$ with $V_{A}$ the Alfven velocity and $r_{s}$ the radius of the $q=1$ surface. In our final dispersion relation, in agreement with the inertial term $\hat{I}$ all terms are normalized by the factor $2 R /\left[\pi\left(B r_{s} \xi\right)^{2}\right]$ with $\mathrm{R}$ the major radius, $\mathrm{B}$ the on-axis magnetic field, and the hat indicates a normalized quantity. All terms in $\delta \hat{W}$ are thus dimensionless and normalized to the shear Alfven frequency. In elongated or high beta equilibria the "minor radius" is defined as $\sqrt{2 \Psi}$ with $\Psi$ the toroidal flux.

The quantities $\delta W_{r}, \delta W_{n}$ are the resonant and nonresonant contributions to $D(\omega)$ due to the high energy particles. The nonresonant contribution is

$$
\delta W_{n}=-\int\left(\vec{\xi}_{\perp} \cdot \vec{\nabla} p\right)\left(\vec{\xi}_{\perp}^{*} \cdot \vec{\kappa}\right) d^{3} x
$$

where $\vec{\xi}_{\perp}$ is the usual displacement introduced in magnetohydrodynamic stability theory, $\vec{k}$ is the curvature

$$
\vec{\kappa}=\frac{\vec{\nabla}\left(B^{2}+2 p\right)}{2 B^{2}}-\frac{\vec{B}(\vec{B} \cdot \vec{\nabla})\left(B^{2}+2 p\right)}{2 B^{4}}
$$


and $p=\int E F d^{3} v$ is the pressure due to the high energy particles

$$
p=\sum_{\sigma} \int \frac{2 \pi B E F}{\sqrt{2 E(1-\mu B / E)}} d \mu d E
$$

with $\mathrm{E}$ the energy, $\mathrm{F}\left(\Psi_{p}, \mathrm{E}, \mu\right)$ the particle distribution function, and $\sigma$ the sign of $v_{\|}$.

Using these expressions the nonresonant part of the kinetic contribution becomes

$$
\delta W_{n}=-2^{3 / 2} \pi^{2} \sum_{\sigma} \int \frac{B E^{1 / 2}}{\sqrt{1-\mu B / E}} H \partial_{\Psi_{p}} F\left(\Psi_{p}, E, \mu\right) \mathcal{J} d \theta d \Psi_{p} d \mu d E
$$

where

$$
H\left(\Psi_{p}, \theta\right)=\left(\vec{\xi}_{\perp}^{*} \cdot \vec{\kappa}\right)\left(\vec{\xi}_{\perp} \cdot \vec{\nabla} \Psi_{p}\right)
$$

The resonant contribution to the dispersion relation is given by (see Appendix A)

$$
\delta W_{r}=2^{7 / 2} \pi^{2} \sum_{\sigma} \int \frac{E^{3 / 2} \bar{J}^{*} Q}{B \sqrt{1-\mu B / E}} \frac{\bar{J}}{\left(\omega_{d}-\omega\right)} \mathcal{J} d \theta d \Psi_{p} d \mu d E
$$

where the bar refers to orbit averaging. In addition

$$
J\left(\Psi_{p}, \theta\right)=(1-\mu B / 2 E)\left(\vec{\xi}_{\perp} \cdot \vec{\kappa}\right) e^{-i n(\zeta-q \theta)}
$$

with $\mathrm{n}$ the toroidal mode number of the mode under consideration. Also

$$
\bar{f}=\frac{1}{2 \pi K_{b}} \int_{-\pi}^{\pi} \frac{f}{B \sqrt{1-\mu B / E}} d \theta
$$

is the bounce average of $\mathrm{f}$, with

$$
K_{b}=\frac{1}{2 \pi} \int_{-\pi}^{\pi} \frac{1}{B \sqrt{1-\mu B / E}} d \theta
$$

and $K_{2}$ is defined similarly, with a factor of $\cos (\theta)$ in the numerator of the integrand (see Appendix B). If necessary (trapped particles), the range of $\theta$ 
is limited to the domain where $1-\mu B / E$ is positive. In large aspect ratio approximation in a circular equilibrium, where $\mathrm{B}$ has a simple $\cos (\theta)$ dependence these functions reduce to lowest order to the usual elliptic integrals, but in a general equilibrium they may be significantly different. The operator $Q=\left[\omega \partial_{E}-(\vec{B} \times \vec{\nabla} S) \cdot \vec{\nabla}\right] F$ with $S=n(\zeta-q \theta)$ reduces to

$$
Q=\omega \partial_{E} F-\partial_{\Psi}, F
$$

Introducing the particle density

$$
d N=4 \pi^{2} \frac{B}{\sqrt{2 E(1-\mu B / E)}} F\left(\Psi_{p}, E, \mu\right) \mathcal{J} d \theta d \Psi_{p} d \mu d E
$$

the nonresonant part of the kinetic contribution becomes

$$
\delta W_{n}=-\int E \bar{H} \frac{\partial_{\Psi_{p}} F}{F} d N
$$

and the resonant part

$$
\delta W_{r}=4 \int E^{2} \frac{\bar{J}^{*}(Q / F) \bar{J}}{\left(\omega_{d}-\omega\right)} d N
$$

In these expressions, the particle distribution $d N$ is an invariant, i.e. equal to the time averaged distribution, so $\mathrm{H}$ has been replaced with its time average. This proceedure is useful for improving the statistics in the Monte-Carlo evaluation. These equations complete the derivation of the dispersion relation. The functions $\vec{\xi}_{\perp} \cdot \vec{\kappa}$ and $\vec{\xi}_{\perp} \cdot \vec{\nabla} \Psi_{p}$ must be supplied either analytically or from a linear MHD code for the equilibrium being considered.

Write the perturbed magnetic field in the form

$$
\delta \vec{B}=\vec{\nabla} \times \alpha \vec{B}=\vec{\nabla} \times(\vec{\xi} \times \vec{B})
$$

Then

$$
\alpha \vec{B}=(\vec{\xi} \times \vec{B})+i \vec{\nabla} G
$$

with $\mathrm{G}$ a gauge. We then have also

$$
B^{2} \vec{\xi}=i \vec{\nabla} G \times \vec{B}
$$


and

$$
B^{2} \alpha=i \vec{B} \cdot \vec{\nabla} G
$$

We then find the two functions of interest to be given by

$$
\vec{\xi} \cdot \vec{\nabla} \Psi_{p}=\frac{i}{\mathcal{J} B^{2}}\left[g \partial_{\theta} G-I \partial_{\zeta} G\right]
$$

and

$$
\vec{\xi} \cdot \vec{\nabla} \theta=\frac{i}{\mathcal{J} B^{2}}\left[-g \partial_{\Psi_{p}} G+\delta \partial_{\zeta} G\right]
$$

Introduce the Fourier expansions

$$
\alpha=\sum_{m} \alpha_{m}\left(\Psi_{p}\right) e^{i(n \zeta-m \theta)}
$$

and

$$
G=\sum_{m} G_{m}\left(\Psi_{p}\right) e^{i(n \zeta-m \theta)}
$$

We then find, on using $\vec{B} \cdot \vec{\nabla}=(1 / \mathcal{J})\left(\partial_{\theta}+q \partial_{\zeta}\right)$

$$
G_{m}=\frac{(g q+I) \alpha_{m}}{m-n q}
$$

and finally

$$
\vec{\xi} \cdot \vec{\nabla} \Psi_{p}=\frac{1}{g q+I} \sum_{m}[g m+n I] G_{m}\left(\Psi_{p}\right) e^{i(n \zeta-m \theta)}
$$

and

$$
\vec{\xi} \cdot \vec{\nabla} \theta=-\frac{1}{g q+I} \sum_{m}\left[i g m \partial_{\Psi_{p}} G_{m}+n \delta G_{m}\right] e^{i(n \zeta-m \theta)}
$$

and

$$
\vec{\xi} \cdot \vec{\kappa}=\vec{\xi} \cdot \vec{\nabla} \Psi_{p}\left[\frac{\partial_{\Psi_{p}} B}{B}+\frac{\partial_{\Psi_{p}} p}{B^{2}}\right] .
$$

Recall that these Fourier expansions are in terms of the straight field line coordinate $\theta$. For use of the cylindrical coordinate $\theta_{c}$ see Appendix B. 


\section{Guiding Center Equations}

Hamiltonian guiding center equations are used to evaluate $\delta W_{r}$ and $\delta W_{n}$. Bounce averages are conveniently converted to time integrals. The guiding center equations are ${ }^{23,22}$

$$
\begin{array}{ll}
\frac{d P_{\zeta}}{d t}=-\partial_{\zeta} \mathcal{H} & \frac{d \zeta}{d t}=\partial_{P_{\zeta}} \mathcal{H} \\
\frac{d P_{\theta}}{d t}=-\partial_{\zeta} \mathcal{H} & \frac{d \theta}{d t}=\partial_{P_{\theta}} \mathcal{H}
\end{array}
$$

where $P_{\theta}=I\left(\rho_{\|}+\alpha\right)+\Psi$ and $P_{\zeta}=g\left(\rho_{\|}+\alpha\right)-\Psi_{p}$ are the canonical momenta with $\rho_{\|}=v_{\|} / B$ and

$$
\mathcal{H}=\frac{1}{2}\left(\frac{P_{\theta}-\Psi}{I}-\alpha\right)^{2} B^{2}+\mu B+\Phi
$$

is the Hamiltonian with $\Phi$ the electric potential and $\alpha$ giving the magnetic perturbation through $\delta \vec{B}=\vec{\nabla} \times \alpha \vec{B}$. Here and in the following we use units given by the on-axis gyro frequency (time), and the major radius (distance). In these units $\rho=\sqrt{2 E}$ is the gyro radius, which is the small parameter in the guiding center approximation. A very important feature of these equations is that motion is given by $B\left(\Psi_{p}, \theta\right)$, the field magnitude only, and real space (metric) quantities such as the Jacobian do not enter.

Substituting $d \theta / d t$ and neglecting terms of higher order in gyroradius it is straightforward to convert a bounce average to a time average

$$
\bar{f}=\frac{1}{T} \int f d t
$$

with $\mathrm{T}$ a bounce (transit) period, or any period much longer than a bounce (transit) period.

\section{Monte-Carlo Particle Distribution}

Write the particle density in terms of the distribution function

$$
d N=F\left(\Psi_{p}, E, \mu\right) d^{3} x d^{3} v
$$


and rewrite the volume element in terms of the magnetic coordinates through

$$
d^{3} x d^{3} v=4 \pi^{2} \sqrt{2 E} \mathcal{J} d \Psi_{p} d \theta d \lambda d E
$$

with the pitch $\lambda= \pm \sqrt{1-\mu B / E}$, the sign given by $v_{\|}$. Normalize through $\beta_{h}$

$$
\beta_{h}=\frac{8 \pi}{B_{0}^{2} V} \int E d N
$$

with $\mathrm{V}$ the plasma volume, and use the Monte-Carlo representation for the density (integrated over $\zeta$ ),

$$
d N=c \sum_{k} \delta\left(\Psi_{p}-\Psi_{p k}\right) \delta\left(\lambda-\lambda_{k}\right) \delta\left(E-E_{k}\right) \delta\left(\theta-\theta_{k}\right) d \Psi_{p} d \lambda d E d \theta
$$

Fixed values of $\Psi_{p}, \lambda, E, \theta$ completely define the orbits. Bounce averaging is carried out numerically, i. e. particles are initiated at particular values of $\Psi_{p}, \theta$ and $\zeta$, and the orbits followed.

For studies of stability with a fixed distribution function it is convenient to directly evaluate $\partial_{\Psi_{p}} F$ in terms of $\mathrm{F}$ rather than integrating by parts as in previous work. ${ }^{11,1}$ Substituting $\mathrm{dN}$ and normalizing $D(\omega)$ through the factor $2 R /\left[\pi\left(B_{0} r_{s} \xi\right)^{2}\right]$ with $\xi$ the amplitude of the $\mathrm{m}=1$ displacement, as done in previous references ${ }^{11,1}$ to simplify the layer term we find

$$
\delta \hat{W}=-\frac{V \beta_{h}}{4 \pi^{2}\left(r_{s} \xi\right)^{2} \sum_{j} E_{j}} \sum_{k}\left[\frac{\partial_{\Psi_{p}} F}{F} E H-\frac{4 E^{2} Q}{F} \frac{\bar{J}^{*} \bar{J}}{\omega_{d}-\omega}\right]_{k} .
$$

The use of Monte-Carlo methods to represent the distribution function leads to a technical difficulty when $\omega$ is in the vicinity of the real axis. The necessary modification of the analysis is discussed in Appendix C.

A rapid means of generating Monte-Carlo distributions and the computing requirements of the code are discussed in Appendix D.

\section{Qualitative Features}

It is convenient to use simple reference examples to serve as numerical test cases, and to illustrate the basic properties of the solutions. For this 
purpose we use the circular large aspect ratio equilibrium, neglect resistive modifications, and restrict the perturbation to consist of a single poloidal harmonic. In the nonresistive limit the dispersion relation reduces to

$$
-i \sqrt{\omega\left(\omega-\omega_{* i}\right)}-\gamma_{I}+\omega_{A} \delta \hat{W}=0 .
$$

In circular flux surface approximation the ideal MHD growth rate $\gamma_{I}$ can be evaluated analytically, ${ }^{25}$ and for a quadratic q profile the result is

$$
\gamma_{I}=\frac{3 \pi \omega_{A} r_{s}^{2}}{R^{2}}[1-q(0)]\left(\beta_{p}^{2}-\frac{13}{144}\right)
$$

with $\omega_{A}$ the shear Alfven frequency and

$$
\beta_{p}=-\frac{2}{B_{\theta}^{2}} \int_{0}^{r_{s}}\left(\frac{r}{r_{s}}\right)^{2} \frac{d p}{d r} d r
$$

where $\mathrm{p}$ is the pressure of the background MHD plasma, not that of the high energy particles.

In the following sections we consider the effect of the high energy particles on the internal kink mode with a perturbation analysis, and discuss in more detail two analytical models, a deeply trapped particle distribution, and a distribution isotropic poloidally and in pitch. These results are not new, but are repeated here because they serve as test cases for the numerical analysis. They also serve to display the great variety of behavior which can be expected depending on the details of the equilibrium and particle distribution.

\section{A. Kink Stabilization, $\gamma_{I}>\omega_{* i} / 2$}

First consider stabilization of the internal kink mode. The initial effect of a high energy population can be understood with a perturbation analysis. The solutions of the dispersion relation have qualitatively different features ${ }^{7}$ according to the relative magnitudes of $\gamma_{I}$ and $\omega_{* i} / 2$. Either term can be larger in a tokamak discharge. First consider $\gamma_{I}>\omega_{* i} / 2$. Writing

$$
\omega=\omega_{* i} / 2+i \sqrt{\gamma_{I}^{2}-\omega_{* i}^{2} / 4}+\Delta
$$

we find to lowest order

$$
\frac{\Delta}{\omega_{A}}=\frac{-i \gamma_{I} \delta \hat{W}}{\sqrt{\gamma_{I}^{2}-\omega_{* i}^{2} / 4}}
$$


and thus Imag $\delta \hat{W}$ positive causes the mode frequency to increase and real $\delta \hat{W}$ positive causes stabilization. Now consider the various contributions to $\delta \hat{W}$. First consider the nonresonant contribution, which is real. For this equilibrium we find (Appendix B)

$$
\delta \hat{W}_{n}=\frac{2^{5 / 2} \pi}{r_{s}^{2}} \sum_{\sigma} \int \frac{E^{1 / 2} \cos (\theta)}{\sqrt{1-\mu B / E}} \partial_{r} F r d r d \theta d \mu d E .
$$

Normally $\partial_{r} F$ is negative, so $\delta \hat{W}_{n}$ is destabilizing for deeply trapped particles (increased pressure gradient in the bad curvature region) and stabilizing for barely trapped and passing particles (increased pressure gradient in the good curvature region), with the largest contribution coming from particles near the trapped-passing boundary, which spend a larger fraction of their time in the good curvature region.

Now consider the resonant contribution. Neglecting the $\omega \partial_{E} F$ term in $Q$, we find

$$
\delta \hat{W}_{r}=-\frac{2^{9 / 2} \pi}{r_{s}^{2}} \sum_{\sigma} \int \frac{E^{3 / 2} \bar{J}^{*} \bar{J} \partial_{r} F}{\left(\omega_{d}-\omega\right) \sqrt{1-\mu B / E}} d r d \theta d \mu d E .
$$

Real $\delta \hat{W}_{r}$ is positive (stabilizing) if $\omega_{d}-\omega_{r}$ is positive and negative (destabilizing) if negative, where $\omega=\omega_{r}+i \gamma$. Typically for deeply trapped high energy particles $\omega_{d}>>\omega$ so they are stabilizing. Low energy trapped particles, barely trapped particles, and passing particles act to destabilize the mode. Imag $\delta \hat{W}_{r}$ is positive for $\gamma>0$ and thus this contribution causes an increase in the frequency of the mode for trapped or passing particles. This frequency change can play an important role in the stabilization process.

\section{B. Kink Stabilization, $\gamma_{I}<\omega_{* i} / 2$}

A similar analysis, for $\gamma_{I}<\omega_{* i} / 2$, shows there are initially two marginally stable modes with frequencies $\omega_{* i} / 2 \pm \sqrt{\omega_{* i}^{2} / 4-\gamma_{I}^{2}}$ and the frequency change is given by

$$
\frac{\Delta}{\omega_{A}}= \pm \frac{\gamma_{I} \delta \hat{W}}{\sqrt{\omega_{* i}^{2} / 4-\gamma_{I}^{2}}}
$$


and thus Imag $\delta \hat{W}$ positive causes destabilization of the high frequency branch and stabilization of the low frequency branch. Real $\delta \hat{W}$ positive causes a frequency increase of the high frequency branch and a frequency decrease of the low frequency branch.

\section{Deeply Trapped Distribution, Kink and Fishbone}

To go beyond a perturbative analysis it is necessary to make further assumptions. First consider a deeply trapped slowing-down particle distribution. Model a particle distribution in r, $\mathrm{E}$, and $\mu$ (i.e. integrated over $\phi$ ) by $d N=c E^{-1} f(r) \delta(\mu-\alpha B) d E r d r d \mu d \theta$, for $E_{0}<E<E_{m}$, with $\mathrm{f}(\mathrm{r})$ the radial density profile normalized through $\int f(r) r d r=1$. We then have

$$
\sum_{\sigma} F=\frac{B_{0}^{2} V \beta_{h} \sqrt{1-\alpha B}}{2^{9 / 2} \pi^{4}\left(E_{m}-E_{0}\right)} E^{-1 / 2} f(r) \delta(\mu-\alpha B) .
$$

Neglecting the $\omega \partial_{E} F$ term in Q, and also using $\omega_{d}=E q K_{2} /\left(r K_{b}\right)$ and approximating $\mathrm{q}$ as 1 everywhere inside the rational surface in the evaluation of $\bar{J}$, we find

$$
\delta \hat{W}=\frac{\beta_{h} B_{0}^{2} V c_{f}}{4 \pi^{2} r_{s}^{2} a} \frac{\omega}{\omega_{d m}} \ln \left(\frac{\omega-\omega_{d m}}{\omega-\omega_{d 0}}\right)
$$

with $\omega_{d m}, \omega_{d 0}$ the maximum and minimum precession frequencies in the distribution, a the minor radius, and $c_{f}$ a shape dependent constant of order unity.

$$
c_{f}=-a \int_{0}^{a} \frac{d f}{d r} r d r
$$

For a parabolic density profile, $f(r) \sim 1-r^{2} / a^{2}, c_{f}=8 / 3$. For a Gaussian profile, $f(r) \sim e^{-(r / h)^{2}}, c_{f}=\sqrt{\pi} a / h$. Here we have approximated the deeply trapped precession frequency $\omega_{d}=E q / r R$ as independent of $\mathrm{r}$. Normalizing $D(\omega)$ through $2 R /\left[\pi\left(B r_{s} \xi_{1}\right)^{2}\right]$ and using $V=2 \pi^{2} a^{2} R$ gives in the nonresistive limit the dispersion relation

$$
-i \sqrt{\omega\left(\omega-\omega_{* i}\right)}-\gamma_{I}+\frac{c_{f} a^{2} R \omega_{A} \beta_{h}}{2 r_{s}^{2} a} \frac{\omega}{\omega_{d m}} \ln \left(\frac{\omega-\omega_{d m}}{\omega-\omega_{d 0}}\right)=0
$$


with the maximum precession frequency $\omega_{d m}$ corresponding to energy $E_{m}$. In the absense of high energy particles, the frequency is given by $\omega=\omega_{* i} / 2+$ $i \sqrt{\gamma_{I}^{2}-\omega_{* i}^{2} / 4}$. This is of course significantly modified if resistive effects are important. Note that the high energy particle contribution is proportional to the mode frequency $\omega$, a consequence of the deeply trapped assumption $(\alpha B \approx 1)$, the simple $\cos (\theta)$ dependence of the magnetic field, the neglect of $\mathrm{m}=2$ contributions, and the neglect of $\mathrm{q}-1$ terms. These are a poor approximations for shaped or high beta equilibria. For comparisons with experiments the full dispersion relation including all such effects may be required.

\section{Deeply Trapped Distribution, $\gamma_{I}>\omega_{* i} / 2$}

Again first consider $\gamma_{I}>\omega_{* i} / 2$. Neglecting $\omega_{* i}, \omega_{d 0}$ and $\gamma_{I}$ and using the total plasma volume to be that contained within the $q=1$ surface, ${ }^{11}$ (i.e. take $\mathrm{a}=r_{s}$ ) this dispersion relation possesses a threshold (the fishbone mode) at

$$
\beta_{h}=\frac{4 \epsilon<\omega_{d}>}{\pi c_{f} \omega_{A}}
$$

with $\epsilon=a / R$, and mode frequency given by the average precession frequency $\left\langle\omega_{d}\right\rangle=\omega_{d m} / 2$. Note thai since $\omega_{d}$ is proportional to particle energy the threshold condition is actually a requirement on hot particle density, not beta.

For $0<\gamma_{I}<.18<\omega_{d}>$ and $\omega_{* i}>0$ there are instead two thresholds, which correspond to the stabilization of the internal kink mode, and the fishbone threshold. ${ }^{4}$ Depending on the values of $\omega_{d 0}$ and $\omega_{* i}$ the internal kink stabilization threshold may take on two very different characters. If stabilization occurs with $\omega$ greater than $\omega_{d 0}$ the logarithm term is complex and $\omega$ must be greater than $\omega_{* i}$. In this case the mode becomes damped for further increase in $\beta_{h}$. If instead stabilization occurs with $\omega$ less than $\omega_{d 0}$ the logarithm term is real and thus $\omega$ must be less than $\omega_{* i}$. For further increase in $\beta_{h}$, the frequency remains real. If instead stabilization occurs with $\omega$ greater than $\omega_{d 0}$ the logarithm term is complex and $\omega$ must be greater than $\omega_{* i}$. In this case the mode becomes damped for further increase in $\beta_{h}$.

For $\omega>>\omega_{d 0}$ we have $\omega>\omega_{* i}$ and the mode frequencies are given by the 
solution of

$$
\sqrt{\omega\left(\omega-\omega_{* i}\right)} \ln \left(\frac{\omega_{d m}}{\omega}-1\right)=\pi \gamma_{I}
$$

The corresponding values of $\beta_{h}$ are given by

$$
\beta_{h}=4<\omega_{d}>\sqrt{1-\frac{\omega_{* i}}{\omega}} \frac{r_{s}^{2} \epsilon}{\pi a^{2} c_{f} \omega_{A}},
$$

a monotone increasing function of frequency.

If instead $\omega_{d m}>>\omega_{d 0}>>\omega$ at threshold, then $\omega<\omega_{* i}$ and threshold is given by the solution of

$$
\sqrt{\omega\left(\omega_{* i}-\omega\right)}-\gamma_{I}+\frac{c_{f} a^{2} R \omega_{A} \beta_{h}}{2 r_{s}^{2} a} \frac{\omega}{\omega_{d m}} \ln \left(\frac{\omega_{d m}}{\omega_{d 0}}\right)=0
$$

and

$$
\frac{\omega_{* i} / 2-\omega}{\sqrt{\omega\left(\omega_{* i}-\omega\right)}}+\frac{c_{f} a^{2} R \omega_{A} \beta_{h}}{2 r_{s}^{2} a \omega_{d m}}\left[\frac{\omega}{\omega_{d 0}}+\ln \left(\frac{\omega_{d m}}{\omega_{d 0}}\right)\right]=0 .
$$

The evolution of the real frequency as $\beta_{h}$ increases further is determined by the first equation alone, and the growth rate remains zero.

For $\gamma_{I}>.18<\omega_{d}>$ there is no threshold. In this case the kink mode branch frequency and growth rate increase as $\beta_{h}$ increases. The fishbone branch instead, remains stable.

\section{Deeply Trapped Distribution, $\gamma_{I}<\omega_{* i} / 2$}

Now consider $\gamma_{I}<\omega_{* i} / 2$. In this case with $\beta_{h}=0$ there are two marginally stable branches of the internal kink mode, with frequencies $\omega_{* i} / 2 \pm \sqrt{\gamma_{I}^{2}-\omega_{* i}^{2} / 4}$. The low frequency branch is stabilized by the high energy particles, and the high frequency branch is destabilized, and has also been suggested ${ }^{12,6}$ as the cause of the fishbone in some discharges. However this branch of the dispersion relation exists even in the absense of the high energy particles, and we will refer to it as the high frequency or ion diamagnetic frequency branch of the internal kink mode. The branch with a threshold at a nonzero value of $\beta_{h}$ and at a frequency given by the average trapped particle precession frequency we will refer to as the fishbone branch. 


\section{Uniform Pitch Distribution}

Also very important is a distribution uniform both poloidally and in pitch, for which the nonresonant contribution to $\delta \hat{W}$ can be evaluated explicitly (see Appendix B). The results are independent of the form of the energy distribution. Writing the distribution in the form $F=f(r) w(E)$ we find for the trapped particle contribution

$$
\delta \hat{W}_{n t}=\frac{\sqrt{2} \beta_{h}}{3 \pi}\left(\frac{a}{r_{s}}\right)^{2} \frac{\int_{0}^{r_{s}} r^{3 / 2}\left[1+\frac{21 r}{15}\right] \frac{d f}{d r} d r}{\int_{0}^{a} r f d r}
$$

where $r_{s}$ is the $q=1$ radius, and $a$ the plasma radius. The total of the trapped and passing particle contributions $\delta \hat{W}_{n}=\delta \hat{W}_{n t}+\delta \hat{W}_{n p}$ is

$$
\delta \hat{W}_{n}=\frac{\beta_{h}}{4}\left(\frac{a}{r_{s}}\right)^{2} \frac{\int_{0}^{r_{s}} r^{2} \frac{d f}{d r} d r}{\int_{0}^{a} r f d r}
$$

Analytic expressions have also been obtained for $\delta \hat{W}_{r}$ for a Maxwellian distribution function, ${ }^{7}$ and we will not repeat these results here. The analytic form for the nonresonant contribution will be used in the next section to test the numerical convergence of the Monte-Carlo integration.

\section{Numerical Results}

In this work we consider only results obtained using the large aspect ratio circular analytic equilibrium. Comparison with experiments and discussion of the effects of plasma shape and beta will be treated in future publications.

The numerical scheme employed involves convergence in the number of Monte-Carlo particles used, which must be controlled to ensure that the desired accuracy has been obtained. We illustrate this convergence with an analytic example which presents more problems numerically than most cases, namely a distribution uniform both poloidally and in pitch. 


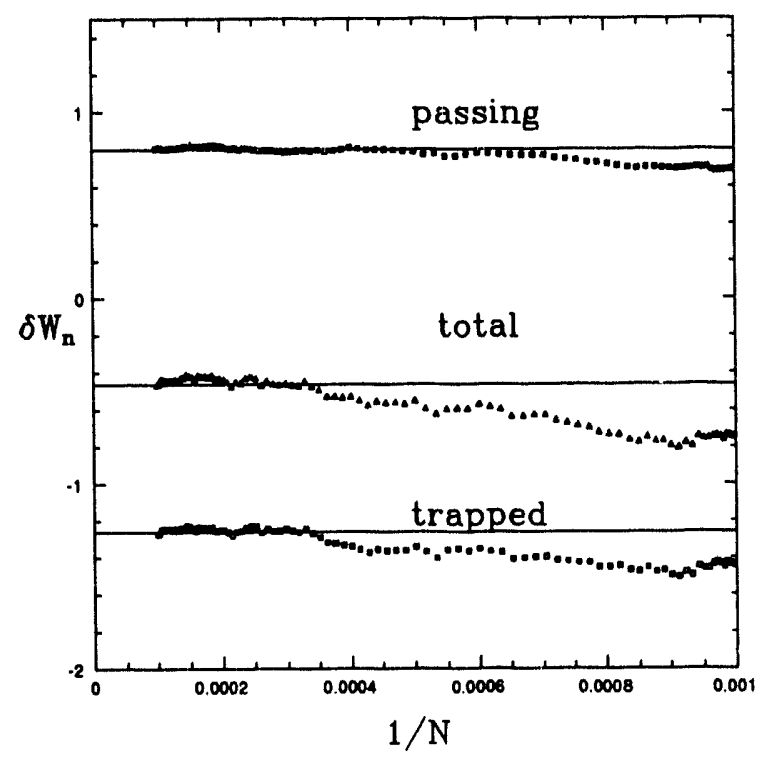

Figure 1: convergence of $\delta W_{n}, \beta_{h}=1$

\section{A. Uniform Pitch Distribution}

In this case the leading order trapped and passing contributions to $\delta \hat{W}_{n}$, of order $\sqrt{R / r}$, have opposite sign and cancel, with the total $\delta \hat{W}_{n}$, of order unity, being significantly smaller than either. Furthermore, much of the contribution of the passing particles is due to particles near the trappedpassing boundary.

We use a large aspect ratio, $\epsilon=.1$ with a large magnetic field strength so that particle excursions away from the flux surface are negligible. The analytic expressions for $\delta \hat{W}_{n}$ are given in section VD. In Fig. 1 are shown the convergence of the trapped, passing and total contributions to $\delta W_{n}$ as a function of the number of particles used. The values shown are for $\beta_{h}=1$. Both the trapped and passing contributions are seen to converge reasonably well to the analytic values (shown as lines) when the number of particles is a few thousand. Statistical fluctuations in $\delta \hat{W}_{n}$ are expected of order $(R / r)^{1 / 2} / \sqrt{N}$ because of the partial cancellation of the trapped and passing contributions. Here the time step was set to require 20 steps per toroidal transit for an on-axis particle with pitch $=1$ and the orbit averages were done 


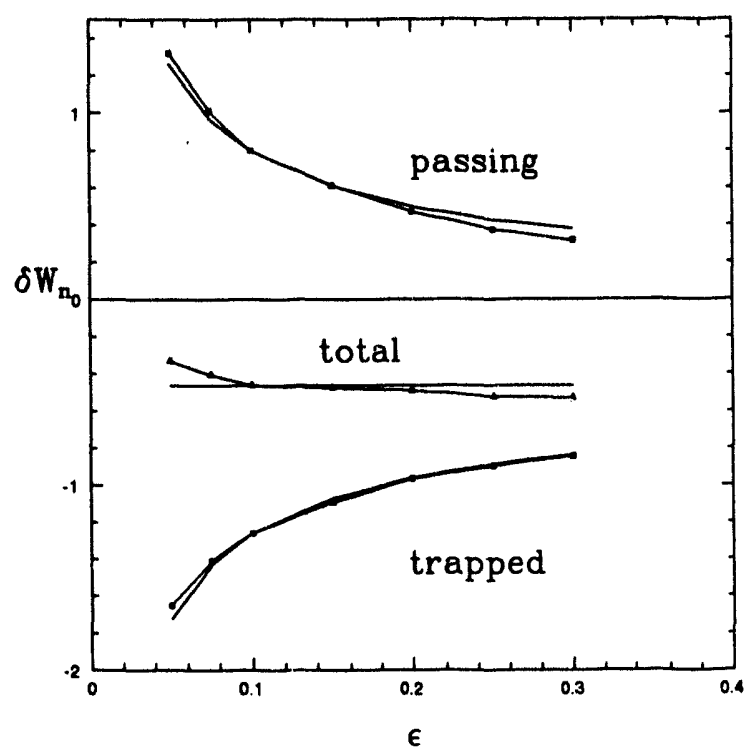

Figure 2: $\delta W_{n}$ vs $\epsilon, \beta_{h}=1$

using 30 such toroidal transit times. For distributions other than uniform in pitch the numerical problems are much less severe, and we conclude that a few thousand particles are generally sufficient. For this example a Gaussian distribution $f(r) \simeq e^{-r^{2} / h^{2}}$ was used, for which

$$
\delta \hat{W}_{n t}=-\frac{2^{5 / 2} \beta_{h} a^{2} \int_{0}^{r_{s} / h} z^{5 / 2}\left[1+\frac{21 h z}{15}\right] e^{-z^{2}} d z}{3 \pi r_{s}^{2} \sqrt{h}\left[1-e^{-a^{2} / h^{2}}\right]}
$$

and

$$
\delta \hat{W}_{n}=-\frac{\beta_{h} a^{2}\left[1-e^{-r_{s}^{2} / h^{2}}\left(1+r_{s}^{2} / h^{2}\right)\right]}{2 r_{s}^{2}\left[1-e^{-a^{2} / h^{2}}\right]} .
$$

The q profile is quadratic in $\mathrm{r}$ with $.8<q<1$., and $r_{s}=a$, and $h=a / 2$. Since the results for the nonresonant contribution are independent of the particle energy distribution, a monoenergetic distribution was used to restrict the particle gyro radius and drift excursions to a single value.

In Fig. 2 is shown the dependence of these terms as a function of the aspect ratio. Also shown are the analytic results. 


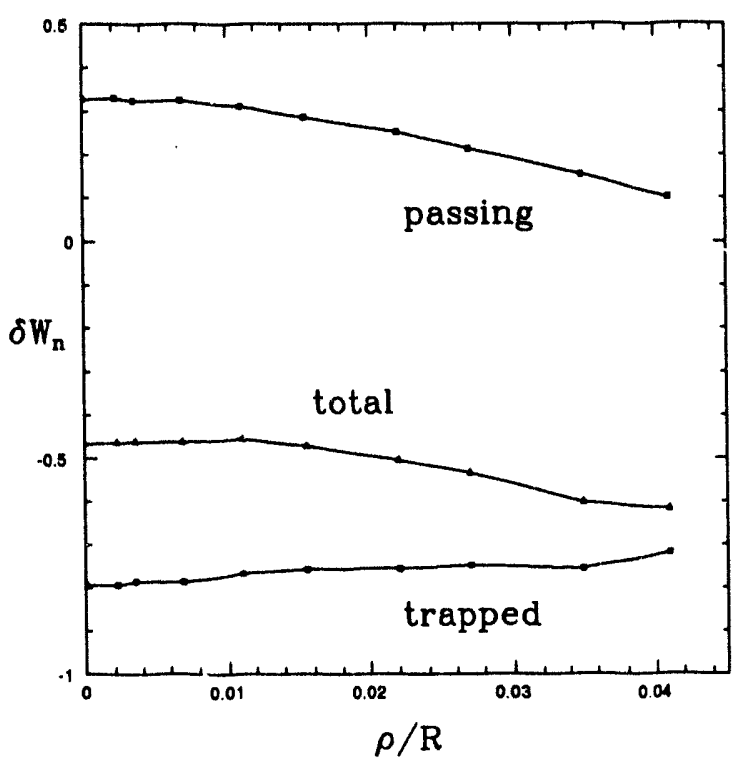

Figure 3: $\delta W_{n}$ vs $\rho / R, \beta_{h}=1$

In Fig. 3 is shown the dependence of these terms as the gyro radius is increased for $\epsilon=.25$. Since the aspect ratio is large, these results extend to values of the gyro radius equal to one fifth of the minor radius, and significant particle loss is encountered. The passing particle contribution is modified the most, as barely passing particles are not well confined, and they contribute the most. The trapped particle term is modified little, since barely trapped particles are lost, and they contribute the least.

\section{B. Deeply Trapped Distribution}

Example results for the deeply trapped slowing down distribution are shown in Figs 4-9.

We produced a trapped distribution by initiating all particles at $\theta= \pm \pi / 4$ with zero parallel velocity. For the equilibrium used $\alpha$ is not exactly constant over the minor radius, varying from 1.01 to 1.1 . If on the other hand, $\alpha$ is kept constant, then all particles inside some radius are passing rather than trapped. The analytic approximations made in evaluating the dispersion relation are not self consistent but can be closely if not exactly duplicated. 


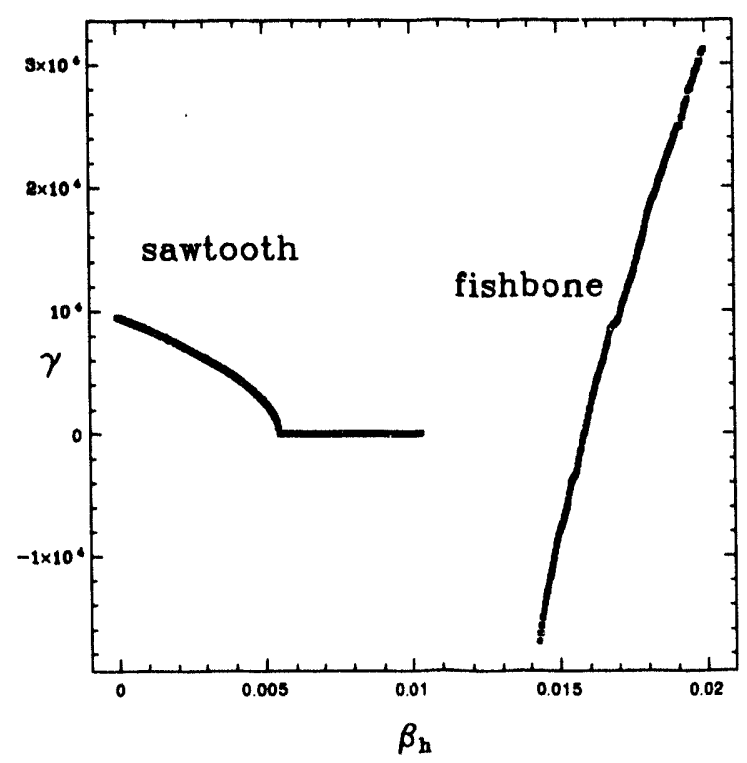

Figure 4: growth rate vs hot particle beta

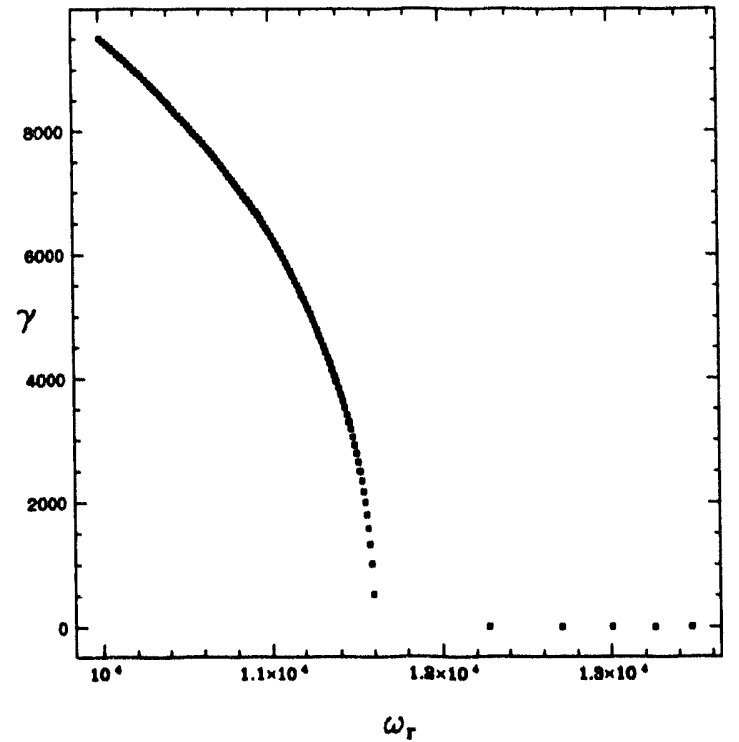

Figure 5: complex frequency plane 
Parameters used are similar to those of previous works, ${ }^{11,1} \mathrm{R}=2.96 \mathrm{M}$, but with very high aspect ratio, $\epsilon=.1$ at the $q=1$ surface, $E_{m}=600 \mathrm{Kev}$, $E_{0}=200 \mathrm{Kev}, \omega_{A}=2 \times 10^{6} \mathrm{sec}^{-1}, \omega_{* i}=2 \times 10^{4} \mathrm{sec}^{-1}, \mathrm{~h}=\mathrm{a}$. The q profile was taken to be quadratic in $\mathrm{r}$, ranging from 0.8 to 1., i.e. the entire plasma volume is taken to be inside the $q=1$ surface, as is consistent with previous calculations, ${ }^{11}$ and there is a single $\mathrm{m}=1$ harmonic. The ideal limit (large S) is used.

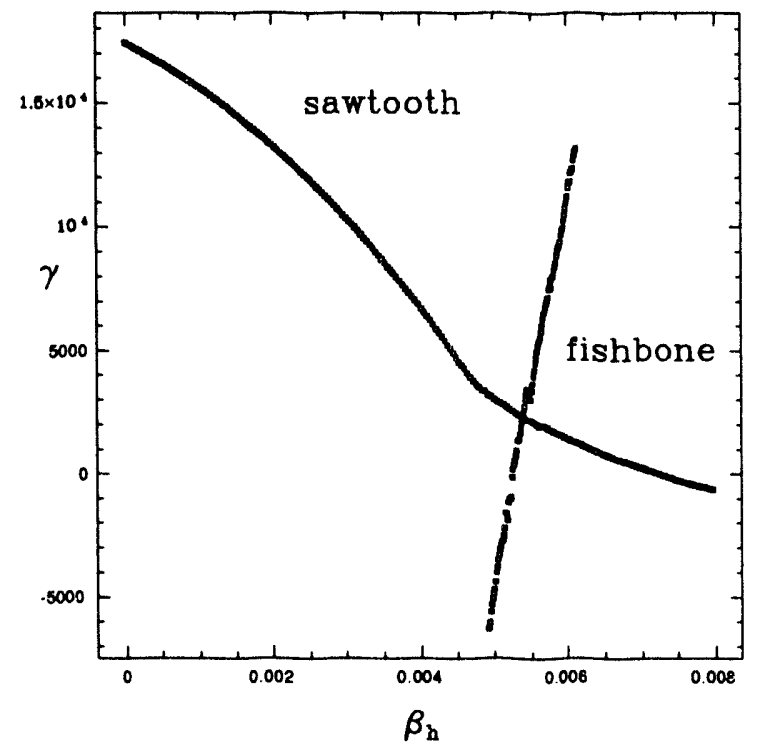

Figure 6: growth rate vs hot particle beta

\section{Deeply Trapped, $\gamma_{I}>\omega_{* i} / 2$}

First consider the case $\gamma_{I}>\omega_{* i} / 2$. We use in this case $\gamma_{I}=1.4 \times 10^{4} / \mathrm{sec}$. In Fig. 4 are shown the growth rates as a function of hot particle beta, for both the sawtooth branch and the fishbone branch. There is a domain of $\beta_{h}$ in which both modes are stable. Analytic expressions for the thresholds are incorrect by as much as 50 ascribed to the approximations made obtaining the analytic expressions, namely neglecting the radial dependence of $\omega_{d}$, using the deeply trapped approximations to evaluate $\mathrm{J}$ and $\mathrm{H}$, and ignoring the q-1 contributions. Details of particle distribution, aspect ratio, and q profile 
can significantly modify the extent of the stable domain and even change qualitative features.

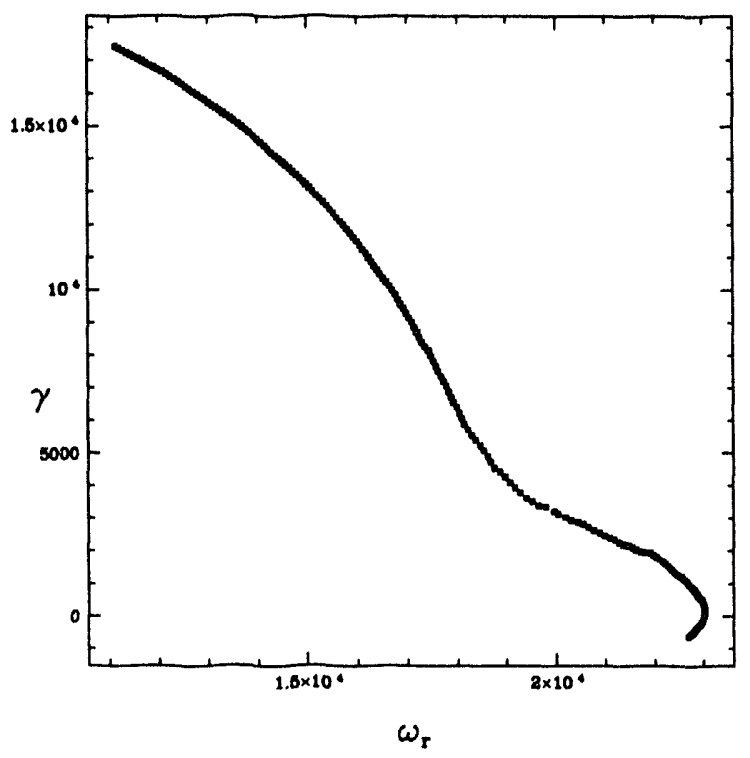

Figure 7: complex frequency plane

In Fig. 5 is shown the trajectory of the sawtooth mode in the complex frequency plane. The real frequency is still significantly less than $\omega_{* i}$ at stabilization, and also less than the minimum precession frequency in the distribution, $\omega_{d 0}$. The increase of the real frequency of the sawtooth mode after stabilization is not relevant. Since $\omega<\omega_{* i}$ the stabilization occurs through the confluence of two roots approaching from the upper and lower half plane. After stabilization the growth rate remains zero, the real frequency of one root then approaches zero and the other increases. The root finder happened to follow the increasing one. The fishbone root approaches $-i \infty$ in the $\omega$ plane as $\beta_{h}$ tends to zero.

In Figs 6,7 are shown results obtained with slightly different parameters. The hot particle distribution has lower energy, equal to $300 \mathrm{Kev}, \omega_{* i}=2.2 \times$ $10^{4} / \mathrm{sec}$ and $\gamma_{I}=2 \times 10^{4} / \mathrm{sec}$. In this case there is no stable domain. The sawtooth stabilization occurs at a frequency larger than $\omega_{* i}$, the presence of which accounts for the circular deviation of the trajectory in the complex frequency plane near threshold. In this case the mode becomes damped. 

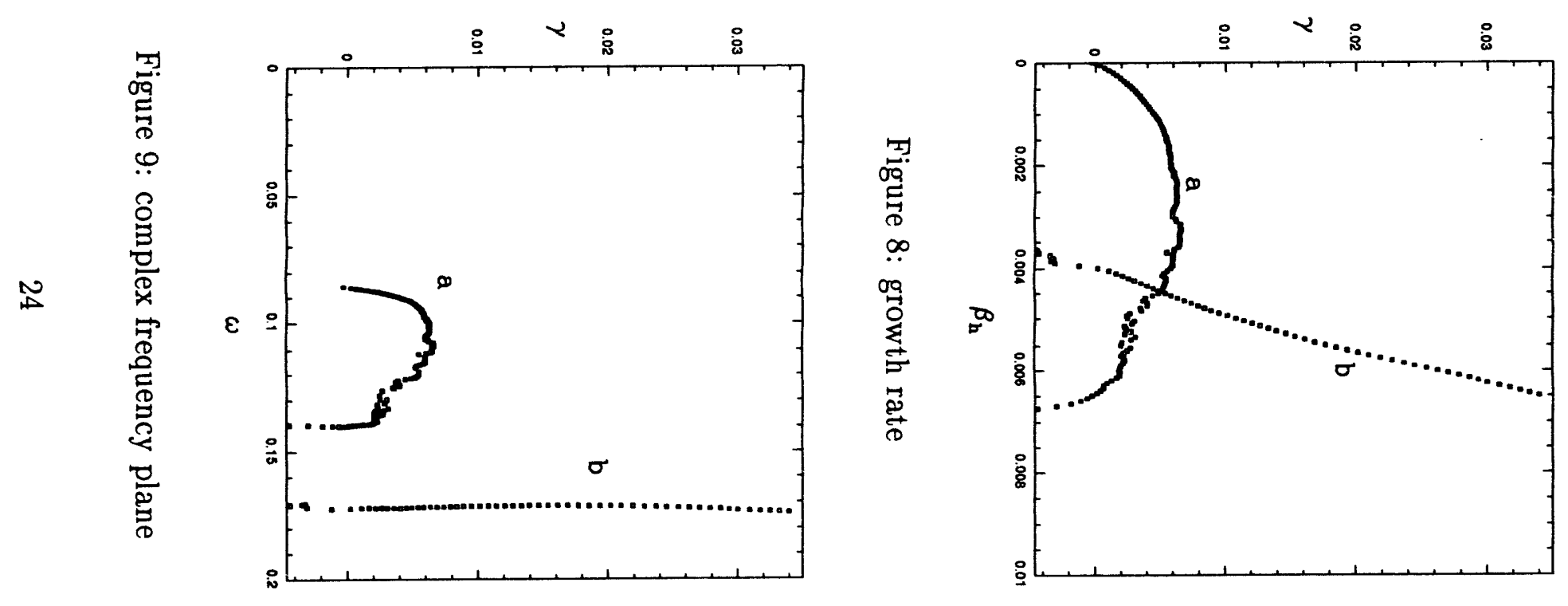


\section{Deeply Trapped, $\gamma_{I}<\omega_{* i} / 2$}

In Figs. 8,9 are shown results with $\gamma_{I}<\omega_{* i} / 2$. We show the growth rates and the trajectories in the complex frequency plane for the destabilized high frequency branch of the kink mode (a) and the precession frequency (fishbone) branch (b). For $\beta_{h}=0$ the kink mode branch solution is marginally stable with real frequency very near the diamagnetic frequency. The fishbone branch is at $-i \infty$. As $\beta_{h}$ increases the kink mode branch is immediately destabilized and its frequency increases. Above threshold the fishbone branch quickly dominates over the kink mode branch, which eventually stabilizes.

In this case the diamagnetic and precession frequencies are well separated, so the modes are easily distinguished, but if these frequencies are very close, it may be difficult to distinguish them in an experiment.

\section{Maxwellian}

Now examine a Maxwellian distribution. As an example we use a fairly realistic tokamak equilibrium, but with circular flux surfaces, with $R=2.28 \mathrm{~m}$, $\mathrm{B}=3.8 \mathrm{~T}$, and an aspect ratio of 3 . The ion diamagnetic frequency is $10^{4}$ /sec, and the Maxwellian (hydrogen) has a temperature of $400 \mathrm{Kev}$. The mode instability is taken to be $\gamma_{I}=1.5 \times 10^{3} / \mathrm{sec}$. Since a uniform pitch distribution is of considerable interest for the analysis of alpha particle effects it is worth examining in detail the various contributions to $\delta W$ in the stabilization process. As noted earlier, the resonant trapped particle contribution dominates both the nonresonant and resonant contributions.

In Figs. 10,11 is shown the stabilization process, achieved at $\beta_{h}=.0012$. In Fig. 12 are shown the real contributions due to $\delta W_{r}$ from the passing and trapped components of the distribution, and the total contribution from the nonresonant part $\delta W_{n}$, as a function of $\beta_{h}$. The resonant passing contribution is destabilizing but smaller than the stabilizing trapped contribution. At stabilization the mode frequency is much less than the ion diainagnetic frequency, and less than one tenth of the average toroidal precession frequency. It is important to note that any estimate of sawtooth stabilization must take into account the frequency dependence of the various contributions. 


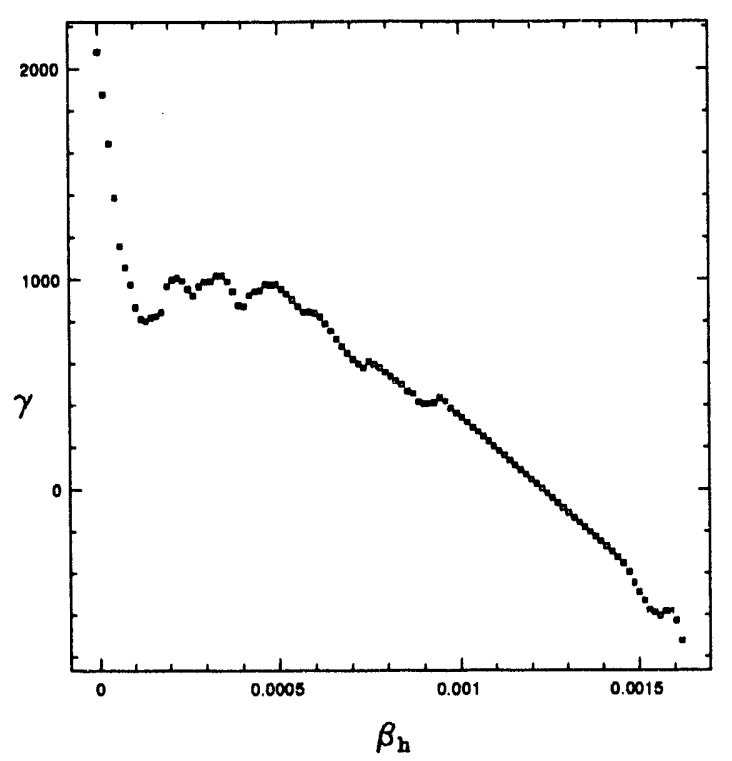

Figure 10: Sawtooth stabilization, Maxwellian

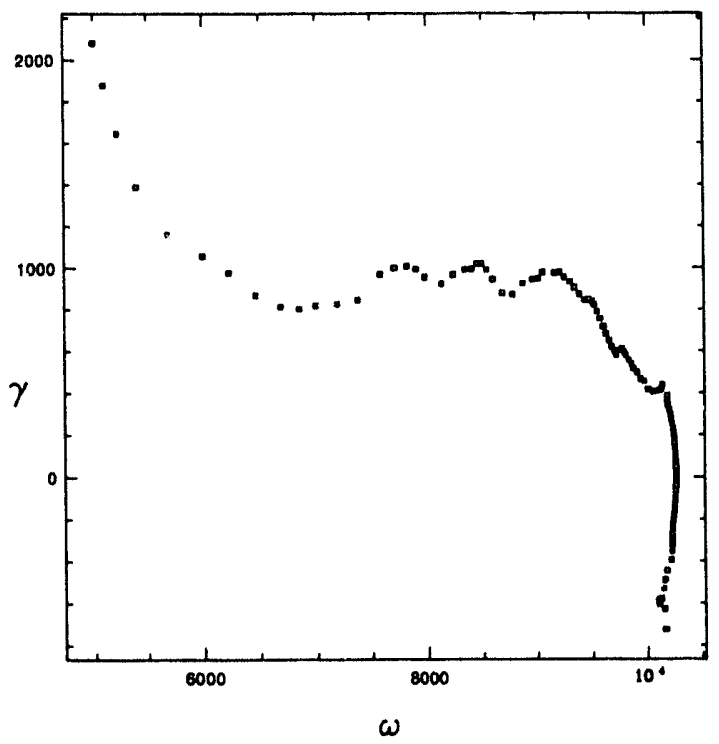

Figure 11: Complex frequency plane, Maxwellian 


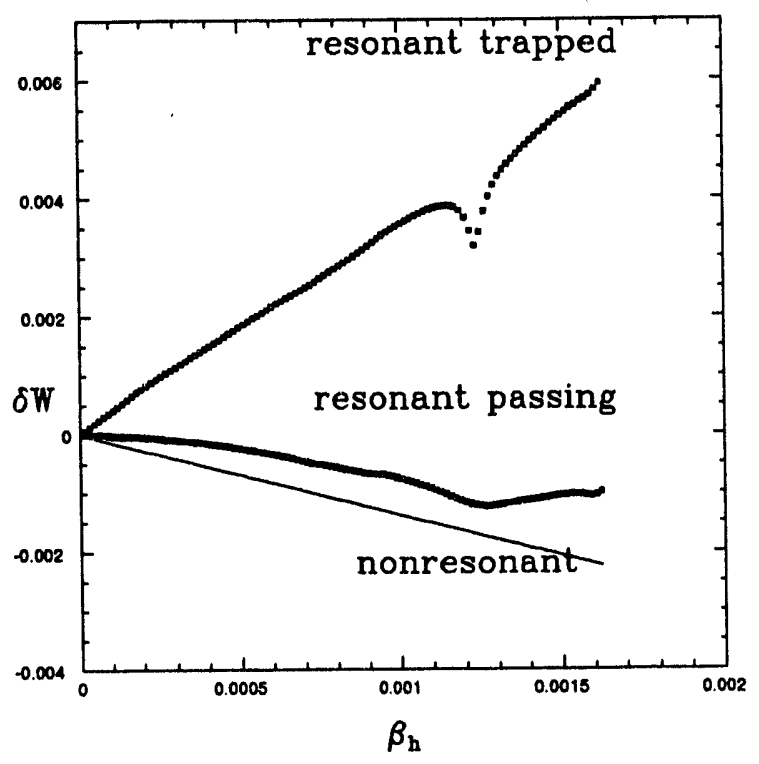

Figure 12: $\delta \hat{W}$, Maxwellian

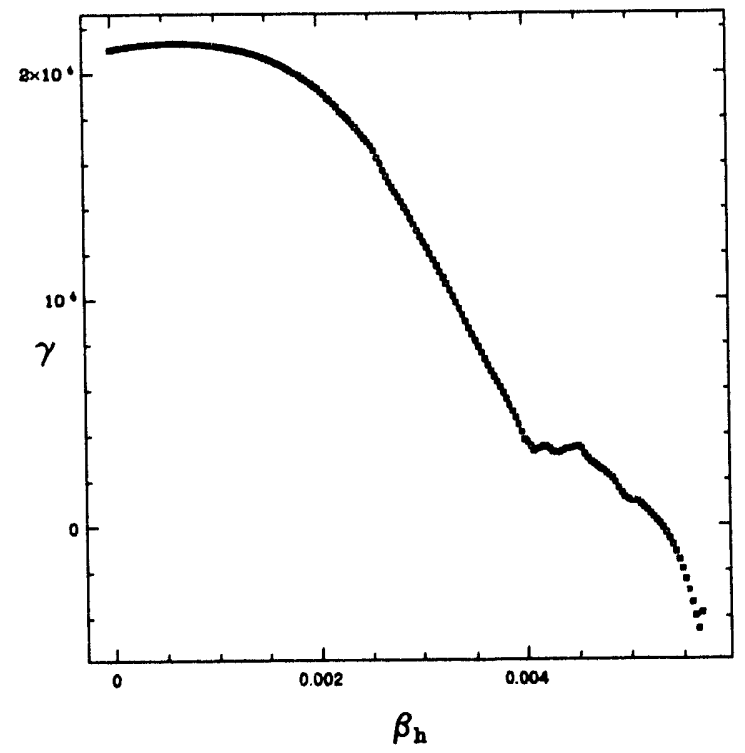

Figure 13: Sawtooth stabilization due to ICRF heating 


\section{ICRF Distribution}

Now examine a model ICRF distribution, with

$$
F \simeq e^{-\left(\frac{s_{1} r}{R}\right)^{2}} e^{-\left[\frac{\theta_{2} E}{E_{m}}+\frac{\left(\mu-\mu_{0}\right)^{2}}{E_{m}^{2} a^{2}}\right]}
$$

truncated at $E_{m}=500 \mathrm{Kev}$, with $s_{1}=11.4, s_{2}=4 ., \mathrm{a}=.05$ and $\mu_{0}$ fixed to localize the center of the bounce tip distribution at the magnetic axis, $\mu_{0}=E / B\left(\Psi_{p}, \pi / 2\right)$. This gives a distribution asymmetry at $\theta=0$ of $\left(2 E_{\|}-E_{\perp}\right) /\left(2 E_{\|}+E_{\perp}\right)=-.5$.

The distribution is somewhat more peaked near the trapped-passing boundary than a Maxwellian, and has many fewer particles in the far passing domain. The equilibrium has $\mathrm{R}=2.28 \mathrm{M}, \mathrm{r}=0.72 \mathrm{M}, \mathrm{B}=3.7 \mathrm{~T}$, and the q profile is $.8<q<2.7$ with $q=q_{0}+c r^{2.3}$. In Fig 13 are shown sample results of stabilization achieved with this distribution function. As is clear from the analysis of the Maxwellian, the stabilization process is completely dominated by the trapped particle contributions.

\section{Conclusion}

A numerical code has been developed for the analysis of the effects of high energy particles on MHD modes. The contribution of the particles is calculated by performing orbit averaging using guiding center equations in analytic or numerically generated equilibria. The high energy particle contribution is used in a linear dispersion relation to solve for the complex mode frequency, which can be followed as a function of equilibrium independent parameters such as diamagnetic frequencies, plasma resistivity, high energy particle beta, etc. A few thousand particles suffice to give an accurate determination of the high energy particle effects. The use of numerical equilibria allows the investigation of the effects of elongation, magnetic well, plasma rotation, etc. The analysis is linear in the MHD phenomena, and the time scale of interest is assumed short compared to changes in the plasma equilibrium. Equilibrium parameters and the linear form of the eigenmode, obtained with a MHD code or with a linear kinetic-MHD code such as NOVA- $\mathrm{K}^{20}$ are input data. The particle distribution is generated by Monte-Carlo methods so that realistic high energy particle distributions are easily realized. Computing requirements are modest, allowing scans of plasma parameters. The 
analysis includes sufficient detail to allow a comparison of kinetic MHD with experiment. The code can be run with mode amplitudes small and fixed, to examine stabilization and destabilization phenomena, or the mode amplitudes can be allowed to grow to study induced particle loss and mode stabilization by this process. Comparison with analytically tractable examples such as deeply trapped and uniform pitch distributions in circular geometry indicate that the method is quite accurate. Future publications will be devoted to the analysis of those high energy particle effects already observed in beam heated and ICRF heated plasmas, and those expected due to the alpha particle distributions in ignited plasmas. 


\section{Appendix A}

We sketch part of the derivation of the resonant part $\delta \hat{W}_{r}$ since most derivations are not given in general magnetic coordinates. For more detail regarding the method see Catto, Tang and Baldwin ${ }^{26}$ and Chen, White and Rosenbluth. ${ }^{11}$ The nonadiabatic part of the perturbed distribution function is given by the solution of

$$
\left(-i \omega+\vec{v}_{\perp} \cdot \vec{\nabla}+v_{\|} \hat{b} \cdot \vec{\nabla}\right) g=2 i E Q(1-\mu B / 2 E) \vec{\xi}_{\perp} \cdot \vec{\kappa}
$$

with $\hat{b}$ the unit magnetic field vector.

This equation is solved perturbatively. To lowest order $v_{\|} \hat{b} \cdot \vec{\nabla} g$ dominates. From axisymmetry we can choose perturbations of a single toroidal harmonic n. Since $\vec{B} \cdot \vec{\nabla}=(1 / \mathcal{J})\left(\partial_{\theta}+q \partial_{\zeta}\right)$ the most general solution to $v_{\|} \hat{b} \cdot \vec{\nabla} g=0$ is then given by

$$
g=g_{0}\left(\Psi_{p}\right) e^{i n(\zeta-q \theta)}
$$

Thus write

$$
g=g_{0}\left(\Psi_{p}\right) e^{i n(\zeta-q \theta)}+g_{1} e^{i n(\zeta-q \theta)}
$$

with $g_{1}$ arbitrary, giving

$$
g_{0}\left[-i \omega+i n \vec{v}_{\perp} \cdot \vec{\nabla}(\zeta-q \theta)\right]+v_{\|} \hat{b} \cdot \vec{\nabla} g_{1}=2 i E Q J
$$

with

$$
J=(1-\mu B / 2 E) \vec{\xi}_{\perp} \cdot \vec{\kappa} e^{-i n(\zeta-q \theta)} .
$$

Now eliminate $g_{1}$ by multiplying by $\mathcal{J} B / v_{\|}$and integrating over the orbit, where $\zeta=q \theta$. Recall that $\mathcal{J} B^{2}$ is independent of $\theta$. The averaged equation then becomes

$$
g_{0}=\frac{2 E Q \bar{J} / F}{-\omega+n \overline{\vec{v}_{\perp} \cdot \vec{\nabla}(\zeta-q \theta)}}
$$


where $\bar{A}$ indicates the bounce average of $\mathrm{A}$ (see Appendix B). Also we know that following the orbit, for any function $f$

$$
\vec{v} \cdot \vec{\nabla} f=\frac{d f}{d t}
$$

and thus we find that

$$
\vec{v}_{\perp} \cdot \vec{\nabla}(\zeta-q \theta)=\frac{d}{d t}(\zeta-q \theta)
$$

However in general ${ }^{27}$ we have

$$
\frac{d}{d t}(\zeta-q \theta)=(\mu-2 E / B) \partial_{\Psi_{p}} B
$$

the time average of which we define to be $\omega_{d}$. This is the toroidal drift for the trapped particles and the cross field drift for the passing particles. Then

$$
g_{0}=\frac{2 E Q \bar{J} / F}{-\omega+\omega_{d}}
$$

where we have used $n=1$.

\section{Appendix B}

Recall that $\theta$ is a straight field line coordinate with a particular choice of Jacobian, not the usual cylindrical coordinate $\theta_{c}$. It is defined so that $\vec{\nabla} \Psi_{p} \cdot(\vec{\nabla} \theta \times \vec{\nabla} \zeta)=B^{2} /(g q+I)$. In cylindrical coordinates instead $\vec{\nabla} \Psi_{p} \cdot\left(\vec{\nabla} \theta_{c} \times\right.$ $\vec{\nabla} \zeta)=1 / X q$. Directly from the Hamiltonian equations, in these straight field line coordinates the integral for the bounce average contains a factor $B \sqrt{1-\mu B / E}$ in the denominator rather than simply $\sqrt{1-\mu B / E}$. Bounce averages of $H\left(\Psi_{p}, \theta\right), J\left(\Psi_{p}, \theta\right)$, and the toroidal precession rate are needed to evaluate $\delta \hat{W}$. In evaluating analytic models it is convenient to perform these bounce averages in the cylindrical coordinate $\theta_{c}$. If $\mathrm{J}$ is expressed in cylindrical coordinates the time integral in these coordinates is given by

$$
\int J_{c} d^{t} \simeq \int J\left(\theta_{c}\right) \frac{d \theta_{c}}{\sqrt{1-\mu B / E}}=\int J\left(\theta_{c}\right) B \frac{d \theta_{c}}{B \sqrt{1-\mu B / E}}
$$


But in this last expression $\theta_{c}$ is a dummy integration variable, and the integral is the time integral of $\mathrm{BJ}$ in straigh: field line coordinates provided the numerical evaluation is done with $B\left(\Psi_{p}, \theta\right)$ taken to be the same function as was used in the analytic model, e.g. If in the analytic model $B\left(\Psi_{p}, \theta_{c}\right)=1-\cos \left(\theta_{c}\right)$ then in the numerical evaluation $B\left(\Psi_{p}, \theta\right)=1-\cos (\theta)$. This can be a more convenient way to evaluate analytic models than expressing everything in terms of straight field line coordinates.

Note that the particle distribution, if uniform spatially, is not uniform in either $\theta_{c}$ or $\theta$, but must be weighted by the appropriate power of $\mathrm{B}$ in the Jacobian. In many cases this is a small correction, but in the case of a distribution which is uniform poloidally and also in pitch, ( a Maxwellian or an alpha particle distribution) the leading order contribution to $\delta \hat{W}_{n}$ is zero, and only the second order survives. by $^{27}$

As a test of Monte Carlo methods we take the lowest order $\vec{\xi}_{\perp}, \vec{\kappa}$, given

$$
\vec{\xi}_{\perp}=\left(\hat{r}-i \hat{\theta}_{c}\right) e^{i\left(\zeta-\theta_{c}\right)}
$$

for $r<r_{s}$, and zero for $r>r_{s}$, and

$$
\vec{\kappa}=-\hat{r} \cos \left(\theta_{c}\right)+\hat{\theta}_{c} \sin \left(\theta_{r}\right) \text {. }
$$

We then find

$$
\vec{\xi}_{\perp} \cdot \vec{\nabla} \Psi_{p}=\frac{q}{r} e^{i\left(\zeta-\theta_{c}\right)}
$$

and

$$
\overrightarrow{\xi_{\perp}} \cdot \vec{\kappa}=-e^{i \zeta},
$$

giving

$$
H=-\frac{q}{r} e^{-i \theta_{c}}
$$

and

$$
J=-(1-\mu B / 2 E) e^{i q \theta_{c}}
$$


For a distribution uniform poloidally and in pitch $\lambda=\sqrt{1-\mu B / E}$, the nonresonant contribution $\delta \hat{W}_{n}$ then takes the form

$$
\delta \hat{W}_{n}=\frac{2^{7 / 2} \pi}{r_{s}^{2} B_{0}^{2}} \int \frac{E^{3 / 2} \cos \left(\theta_{c}\right)}{B} \partial_{r} F r d r d \theta_{r} d \lambda d E
$$

with $\mathrm{F}$ a function of $\mathrm{E}$ and $\mathrm{r}$ only. Write $\mathrm{F}$ in the form $F=w(E) f(r)$ and normalize through $\beta_{h}$, giving to two orders in inverse aspect ratio

$$
\delta \hat{W}_{n}=\frac{\beta_{h} a^{2}}{8 \pi r_{s}^{2}} \frac{\int \frac{\cos \left(\theta_{c}\right)}{B} \partial_{r} f r d r d \theta_{c} d \lambda}{\int f r d r} .
$$

The trapped particle contribution is given by the restriction $|\lambda|<$ $\sqrt{1-B(r, \theta) / B(r, \pi)}$ and the total contribution by $|\lambda|<1$. The total then becomes

$$
\delta \hat{W}_{n}=\frac{\beta_{h} a^{2}}{4 r_{*}^{2}} \frac{\int \partial_{r} f r^{2} d r}{\int f r d r}
$$

and, substituting $w=\sin (\theta / 2)$ to facilitate the integration, the trapped particle contribution is

$$
\delta \hat{W}_{n t}=\frac{\beta_{h} a^{2}}{3 \pi r_{s}^{2}} \frac{\int \sqrt{2 r}\left[1+\frac{21 r}{15}\right] \partial_{r} f r d r}{\int f r d r}
$$

with $\delta \hat{W}_{n}=\delta \hat{W}_{n t}+\delta \hat{W}_{n p}$.

\section{Appendix C}

If $\omega=\omega_{r}+i \gamma$ is in the vicinity of the real axis and there are MonteCarlo points near $\omega_{r}$ the discreteness of the sum in the representation of the resonant part of the dispersion relation can give large errors. Consider the discrete representation of an integral

$$
\sum \frac{g\left(\omega_{d k}\right)}{\omega_{d k}-\omega}=\int \frac{d n}{d \omega_{d}} \frac{g\left(\omega_{d}\right)}{\left(\omega_{d}-\omega\right)} d \omega_{d}
$$


with $d n / d \omega_{d}$ the (smooth probability) density of points in the interval $d \omega_{d}$. The discrete sum clearly fails to give an accurate representation of the integral when $\gamma<d \omega_{d} / d n$, the discrete particle spacing on the real axis in the vicinity of $\omega_{r}$. Since we are interested in mode stabilization and destabilization it is necessary to modify the numerical algorithm for the evaluation of the sum when $\omega$ is near the real axis. For this purpose construct a local Taylor expansion for $g\left(\omega_{d}\right)$ in the vicinity of $\omega_{r}$, i.e. let

$$
g\left(\omega_{d}\right)=a+b \omega_{d}
$$

in a domain $\mathrm{D}$ defined by $\omega_{1}<\omega_{d}<\omega_{2}$ with $\omega_{1}=\omega_{r}-D / 2, \omega_{2}=\omega_{r}+D / 2$. The domain $\mathrm{D}$ must be chosen sufficiently large to contain many Monte-Carlo points. Fix a,b using the local integrals

$$
\begin{gathered}
\sum g\left(\omega_{d k}\right)=\int \frac{d n}{d \omega_{d}} g\left(\omega_{d}\right) d \omega_{d l}, \\
\sum \omega_{d k} g\left(\omega_{d k}\right)=\int \frac{d n}{d \omega_{d}} \omega_{d} g\left(\omega_{d}\right) d \omega_{d} .
\end{gathered}
$$

We then find an expression which is well behaved on the real axis and can be analytically continued to the lower half plane,

$$
\sum_{k} \frac{g\left(\omega_{d k}\right)}{\omega_{d k}-\omega} \rightarrow \sum_{k \neq D} \frac{g\left(\omega_{d k}\right)}{\omega_{d k}-\omega}+N b+\frac{N(a+b \omega)}{D}\left[\log \left(\frac{\omega_{2}-\omega}{\omega-\omega_{1}}\right)+i \pi\right]
$$

with $\mathrm{N}$ the number of Monte-Carlo points in the domain D. For $\gamma>0$ the last term vanishes as $\gamma \rightarrow \infty$. For $\gamma<0$ the last term approaches $2 \pi i g(\omega) d n / d \omega_{d}$ for $\gamma \rightarrow-\infty$, but this expression is valid only in the vicinity of the real axis because of the use of the Taylor expansion for $g$. Since we are only interested in threshold phenomena, and not in following roots of the dispersion relation far below threshold, this procudure is quite adequate, and allows an accurate investigation of threshold phenomena.

\section{Appendix D}

Introduce the pitch $\lambda=v_{\|} / v=\sqrt{1-\mu B / E}$. The particle density is then 


$$
d n=4 \pi^{2} \sqrt{2 E} F(E, \mu, r, \theta) \mathcal{J} d \lambda d E d \theta d \Psi_{p} .
$$

A Monte-Carlo distribution can be constructed by selecting points uniformly distributed in $\lambda, E, \theta, \Psi_{p}$ and accepting them as part of the distribution with probability $4 \pi^{2} \sqrt{2 E} F(E, \mu, r, \theta) \mathcal{J}$. (Directly calculating the distribution using the variable $\mu$ is not satisfactory because of the resulting factor of $\sqrt{1-\mu B / E}$ appearing in the denominator of the probability, which is unbounded.) For most plasma physics applications the distribution function has exponential or near exponential form in both energy and radius. This need not be a large aspect ratio approximation. The radius can refer to the flux value on the midplane $\theta=0$. It is then convenient to perform this part of the Monte-Carlo generation analytically. Suppose the distribution to have the form

$$
F=g(E, \mu, r, \theta) e^{-r^{2} / h^{2}} e^{-E / T}
$$

with $g(E, \mu, r, \theta)$ a relatively slowly varying function of its arguements. The particle density is then

$$
d n \simeq \sqrt{E} g(E, \mu, r, \theta) e^{-r^{2} / h^{2}} e^{-E / T} \mathcal{J} \frac{d \Psi_{p}}{d r^{2}} d \lambda d E d \theta r d r .
$$

Introduce the variables $1-z=e^{-r^{2} / h^{2}}$ and $1-w=e^{-E / T}$, giving

$$
d n \simeq \sqrt{E} g(E, \mu, r, \theta) \mathcal{J}\left(d \Psi_{p} / d r^{2}\right) d \lambda d z d \theta d w
$$

and thus the distribution is formed by selecting points uniformly distributed in $\theta, \lambda \mathrm{z}, \mathbf{w}$ and then making the corresponding $E=-T \log (1-w)$, $r^{2}=-h^{2} \log (1-z), \mu=E\left(1-\lambda^{2}\right) / B$ part of the distribution with probability $\sqrt{E} g(E, \mu, r, \theta) \mathcal{J} d \Psi_{p} / d r^{2}$. The weak dependence of this function ensures a Monte-Carlo generation of the distribution with a small amount of computing. 
We find typically that an analysis of a given equilibrium and particle distribution requires a few thousand Monte-Carlo particles and bounce averaging for 20 or 30 transit times, with a transit time the time for one toroidal transit of an on-axis particle with pitch $=1$. Performing the bounce averages and tracking one or more roots of the dispersion relation as a function of some parameter such as the high energy particle beta, plasma resistivity or diamagnetic frequency typically requires 40 seconds of CRAY time. Thus it is relatively easy to perform scans of parameter values.

\section{Acknowledgement}

This work was partly supported by the U.S.D.o.E. under contract number DE-AC02-76-CHO3073. One of the authors (R.B.W.) is grateful for the hospitality shown him while visiting Cadarache, where much of this work was completed, and for helpful discussions with Xavier Garbet and Maxime Zabiego. We are also grateful to Liu Chen for many useful discussions. 


\section{References}

${ }^{1}$ R. B. White, L. Chen, F. Romenelli, and R. Hay, Phys. Fluids B 28, 278 (1985).

${ }^{2}$ R. B. White, P. H. Rutherford, P. Colstock, and M. N. Bussac, Phys. Rev. Lett. 60, 2038 (1988).

${ }^{3}$ B. Coppi, R. J. Hastie, S. Migliuolo, F. Pegoraro, and F. Porcelli, Phys. Lett. A 132, 267 (1988).

${ }^{4}$ R. B. White, M. N. Bussac, and F. Romanelli, Phys. Rev. Lett. 62, 539 (1989).

${ }^{5}$ L. Chen, R. B. White, G. Rewoldt, P. Colstock, P. H. Rutherford, Y. P. Chen, F. J. Ke, S. T. Tsai, and M. N. Bussac, Proceedings of the Twelfth International Conference on Plasma Physics and Controlled Nuclear Fusion Research, International Atomic Energy Agency, Vienna, 1989 2, 77.

${ }^{6}$ F. Pegoraro, F. Porcelli, B. Coppi, P. Detragiache, and S. Migliuolo, Proceedings of the Twelfth International Conference on Plasma Physics and Controlled Nuclear Fusion Research, International Atomic Energy Agency, Vienna, 1989 2, 243.

${ }^{7}$ R. B. White, F. Romanelli, and M. N. Bussac, Phys. Fluids B 4, 745 (1990).

${ }^{8}$ A. B. Mikhailovskii, jetp 41, 890 (1975).

${ }^{9}$ M. N. Rosenbluth and P. H. Rutherford, Phys. Rev. Lett. 34, 1428 (1975).

${ }^{10}$ K. T. Tsang, D. J. Sigmar, and J. C. Whitson, Phys. Fluids 24, 1508 (1981).

${ }^{11}$ L. Chen, R. B. White, and M. N. Rosenbluth, Phys. Rev. Lett. 52, 1122 (1984).

${ }^{12}$ B. Coppi and F. Porcelli, Phys. Rev. Lett. 57, 2272 (1986).

${ }^{13}$ C. Z. Cheng, L. Chen, and M. S. Chance, Ann. Phys. NY 161, 21 (1984). 
${ }^{14} \mathrm{~S}$. T. Tsai and L. Chen, Phys. Fluids B 5, 3284 (1993).

${ }^{15}$ R. B. White, R. Goldston, K. McGuire, A. A. Boozer, D. A. Monticello, and W. Park, Plasma Phys. Controlled Nucl. Research 3, 391 (1983).

${ }^{16}$ D. J. Sigmar, C. T. Hsu, R. B. White, and C. Z. Cheng, Phys. Fluids B 4 (6), 1506 (1992).

${ }^{17} \mathrm{JET}$ Team, Proceedings of the Eleventh International Conference on Plasma Physics and Controlled Nuclear Fusion Research, International Atomic Energy Agency, Vienna, 1987 1, 449.

${ }^{18}$ C. Phillips, J. Hosea, E. Marmar, M. W. Phillips, J. Snipes, J. Stevens, J. Terry, J. R. Wilson, M. Bell, M. Bitter, R. Boivin, C. Bush, C. Z. Cheng, D. Darrow, e. Fredrickson, R. Goldfinger, G. W. Hammett, K. Hill, D. Hoffman, W. Houlberg, H. Hsuen, M. Hughes, D. Jassby, D. McCune, K. McGuire, Y. Nagayama, D. K. Owens, H. Park, A. Ramsey, G. Schilling, J. Schivell, D. N. Smithe, B. Stratton, E. Synakowski, G. Taylor, H. Towner, R. White, and S. Zweben, Phys. Fluids B 4, 2155 (1992).

${ }^{19}$ W. Park, S. Parker, H. Biglari, M. Chance, L. Chen, C. Z. Cheng, T. S. Hahm, W. W. Lee, R. Kulsrud, D. Monticello, L. Sugiyma, and R. B. White, Phys. Fluids B 4, 2033 (1992).

${ }^{20} \mathrm{C}$. Z. Cheng, Phys. Reports 211, 1 (1992).

${ }^{21}$ A. H. Boozer, Phys. Fluids 29, 4123 (1986).

${ }^{22}$ R. B. White and M. S. Chance, Phys. Fluids 27, 2455 (1984).

${ }^{23}$ R. B. White, Phys. Fluids B 2, 845 (1990).

${ }^{24}$ G. Ara, B. Basu, B. Coppi, G. Laval, M. N. Rosenbluth, and B. V. Waddell, Ann Phys. 112, 443 (1975).

${ }^{25}$ M. N. Bussac, R. Pellat, D. Edery, and J. L. Soule, Phys. Rev. Lett. 35, 1638 (1975).

${ }^{26}$ P. J. Catto, W. M. Tang, and D. E. Baldwin, Plasma Phys. 23, 639 (1981).

${ }^{27}$ R. B. White, Theory of Tokamak Plasma, North Holland, 1989. 
Dr. F. Paoloni, Univ. of Wollongong, AUSTRALIA

Prof. M.H. Brennan, Univ. of Sydney, AUSTRALIA

Plasma Research Lab., Australian Nat. Univ., AUSTRALIA

Prof. I.R. Jones, Flinders Univ, AUSTRALIA

Prof. F. Cap, Inst. for Theoretical Physics, AUSTRIA

Prof. M. Heindler, Institut für Theoretische Physik, AUSTRIA

Prol. M. Goossens, Astronomisch Instituut, BELGIUM

Ecole Royale Militaire, Lab. de Phy. Plasmas, BELGIUM

Commission-European, DG. XII-Fusion Prog., BELGIUM

Prot. R. Bouciqué, Rijksuniversiteit Gent, BELGIUM

Dr. P.H. Sakanaka, Instituto Fisica, BRAZIL

Prof. Dr. I.C. Nascimento, Instituto Fisica, Sao Paulo, BRAZIL Instituto Nacional De Pesquisas Espaciais-INPE, BRAZIL Documents Office, Atomic Energy of Canada Lid., CANADA Ms. M. Morin, CCFMTokamak de Varennes, CANADA Dr. M.P. Bachynski, MPB Technologies, Inc., CANADA Dr. H.M. Skarsgard, Univ. of Saskatchewan, CANADA Prof. J. Teichmann, Univ. of Montreal, CANADA Prof. S.R. Sreenivasan, Univ. of Calgary, CANADA Prof. T.W. Johnston, INRS-Energie, CANADA

Dr. R. Boiton, Centre canadien de fusion magnétique, CANADA

Dr. C.R. James,. Univ. of Alberta, CANADA

Dr. P. Lukác, Komenskèho Universzita, CZECHO-SLOVAKIA The Librarian, Cutham Laboratory, ENGLAND Library, R61, Ruthertord Appleton Laboratory, ENGLAND Mrs. S.A. Hutchinson, JET Library, ENGLAND Dr. S.C. Shama, Univ of South Pacific. FIJI ISLANDS P. Mähönen, Univ. of Helsinki, FINLAND Prot. M.N. Bussac, Ecole Polytechnique., FRANCE C. Mouttet, Lab. de Physique des Milieux lonisés, FRANCE J. Radet, CEN/CADARACHE - Bat 506, FRANCE Prot. E. Economou, Univ. of Crete, GREECE Ms. C. Rinni, Univ. of loannina, GREECE Preprint Library, Hungarian Academy of Sa., HUNGARY Dr. B. DasGupta, Saha Inst. of Nuclear Physics, INDIA Dr. P. Kaw, Inst. for Plasma Research, INDIA Dr. P. Rosenau, Israel Inst. of Technology, ISRAEL Librarian, Intemational Center for Theo Physics, ITALY Miss C. De Palo, Associazione EURATOM-ENEA, ITALY Dr. G. Grosso, Istituto di Fisica del Plasma, ITALY Prof. G. Rostangni, Istituto Gas Ionizzati Del Cnr, ITALY
Dr. H. Yamato, Toshiba Res \& Devel Center, JAPAN

Prof. I. Kawakami, Hiroshima Univ., JAPAN

Prof. K. Nishikawa, Hiroshima Univ., JAPAN

Librarian, Naka Fusion Research Establishment, JAERI, JAPAN

Director, Japan Atomic Energy Research Inst., JAPAN

Prof. S. Itoh, Kyushu Univ., JAPAN

Research Info. Ctr., National Instit. for Fusion Science, JAPAN

Prof. S. Tanaka, Kyoto Univ., JAPAN

Library, Kyoto Univ., JAPAN

Prof. N. Inove, Univ. of Tokyo, JAPAN

Secretary, Plasma Section, Electrotechnical Lab., JAPAN

S. Mori, Technical Advisor, JAERI, JAPAN

Dr. O. Mitarai, Kumamoto Inst. of Technology, JAPAN

Dr. G.S. Lee, Korea Basic Sci. Ctr., KOREA

J. Hyeon-Sook, Korea Alomic Energy Research Inst., KOREA

D.I. Choi, The Korea Adv. Inst. of Sci. \& Tech., KOREA

Prof. B.S. Liley, Univ. of Waikato, NEW ZEALAND

Inst of Physics, Chinese Acad Sci PEOPLE'S REP. OF CHINA Library, Inst. of Plasma Physics, PEOPLE'S REP. OF CHINA Tsinghua Univ. Library, PEOPLE'S REPUBLIC OF CHINA Z. Li, S.W. Inst Physics, PEOPLE'S REPUBLIC OF CHINA Prof. J.A.C. Cabral, Instituto Superior Tecnico, PORTUGAL Prof. M.A. Hellberg. Univ of Natal, S. AFRICA Prot. D.E. Kim, Pohang Inst. of Sci. \& Tech., SO. KOREA Prot. C.I.E.M.A.T, Fusion Division Library, SPAIN Dr. L. Stenflo, Univ, of UMEA, SWEDEN Library, Royal Inst. of Technology, SWEDEN Prof. H. Wilhelmson, Chalmers Univ. of Tech., SWEDEN Centre Phys. Des Plasmas, Ecole Polytech, SWITZERLAND Bibliotheek, Inst. Voor Plasma-Fysica, THE NETHERLANDS Asst. Prot. Dr. S. Cakir, Middle East Tech. Univ., TURKEY Dr. V.A. Glukhıkh,Sci. Res. Inst. Electrophys.I Apparatus, USSR Dr. D.D. Ryutov, Siberian Branch of Academy of Sci., USSR Dr. G.A. Eliseev, I.V. Kurchatov Inst., USSR Librarian, The Ukr.SSR Academy of Sciences, USSR Dr. L.M. Kovrizhnykh, Inst. of General Physics, USSR Kerntorschungsanlage GmbH, Zentralbibliothek, W. GERMANY Bibliothek, Inst. Für Plasmaforschung, W. GERMANY Prof. K. Schindler, Ruhr-Universitát Bochum, W. GERMANY Dr. F. Wagner, (ASDEX), Max-Planck-Institut, W. GERMANY Librarian, Max-Planck-Institut, W. GERMANY 

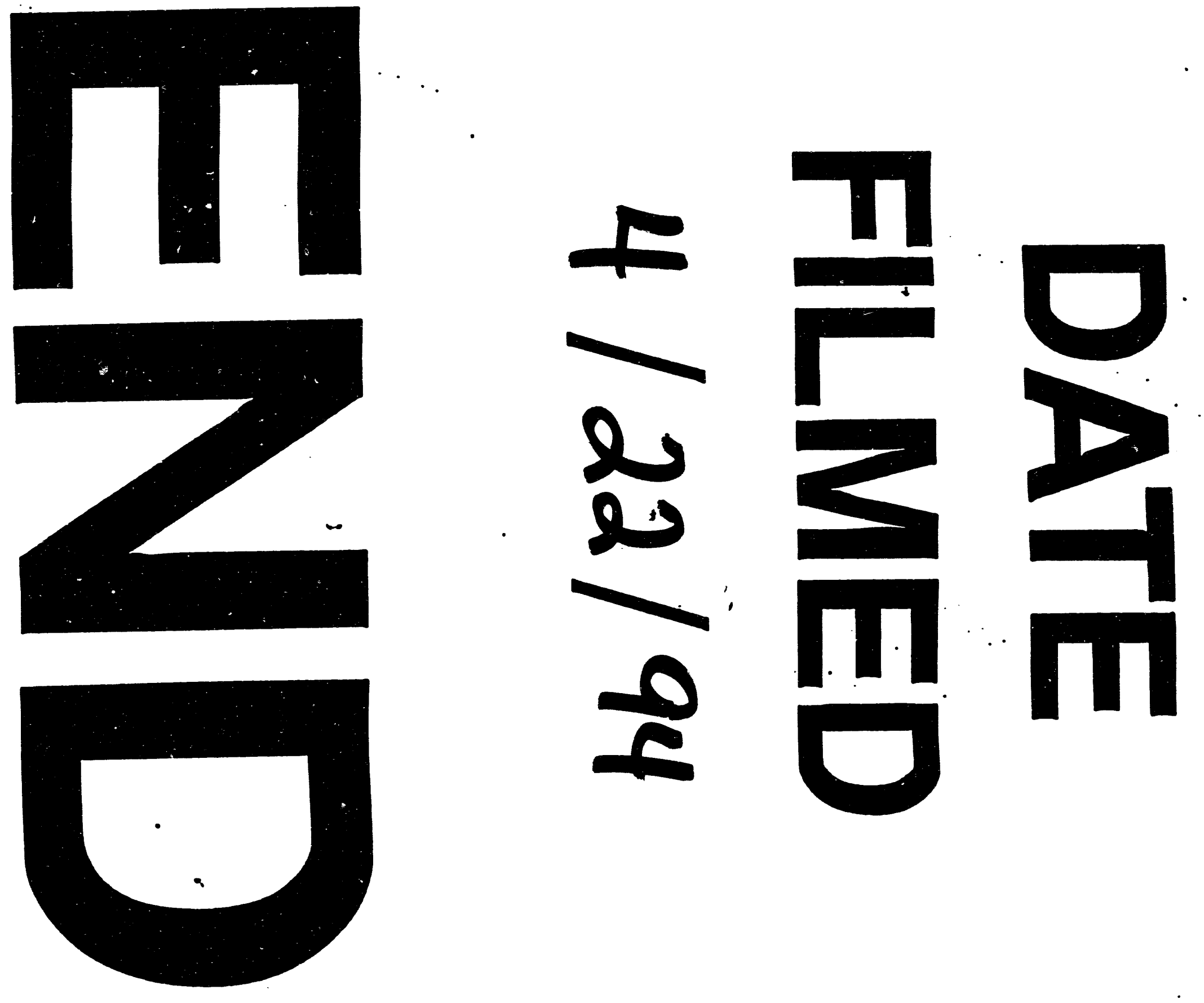


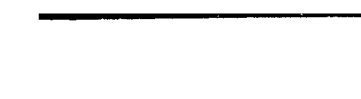

$$
\text { (1) }
$$ \\ $\overline{c o s}$}

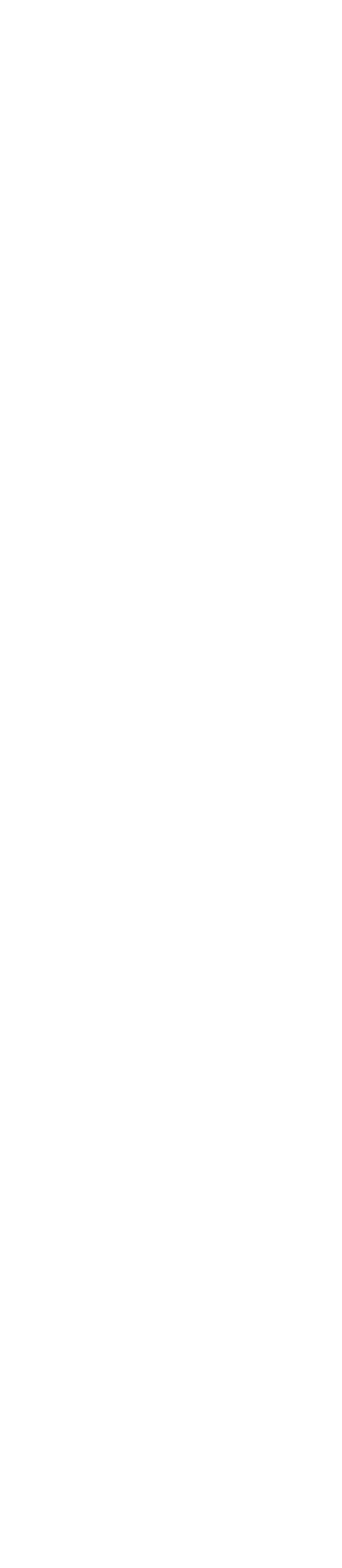 \\ (2)}

